DESY-02-072

May 2002

\title{
Measurement of proton-dissociative diffractive photoproduction of vector mesons at large momentum transfer at HERA
}

\author{
ZEUS Collaboration
}

\begin{abstract}
Diffractive photoproduction of vector mesons, $\gamma p \rightarrow V Y$, where $Y$ is a protondissociative system, has been measured in $e^{+} p$ interactions with the ZEUS detector at HERA using an integrated luminosity of $25 \mathrm{pb}^{-1}$. The differential cross section, $\mathrm{d} \sigma / \mathrm{d} t$, is presented for $-t<12 \mathrm{GeV}^{2}$, where $t$ is the square of the four-momentum transferred to the vector meson. The data span the range in photon-proton centre-of-mass energy, $W$, from $80 \mathrm{GeV}$ to $120 \mathrm{GeV}$. The $t$ distributions are well fit by a power law, $\mathrm{d} \sigma / \mathrm{d} t \propto(-t)^{-n}$. The slope of the Pomeron trajectory, measured from the $W$ dependence of the $\rho^{0}$ and $\phi$ cross sections in bins of $t$, is consistent with zero. The ratios $\mathrm{d} \sigma_{\gamma p \rightarrow \phi Y} / \mathrm{d} t$ to $\mathrm{d} \sigma_{\gamma p \rightarrow \rho^{0} Y} / \mathrm{d} t$ and $\mathrm{d} \sigma_{\gamma p \rightarrow J / \psi Y} / \mathrm{d} t$ to $\mathrm{d} \sigma_{\gamma p \rightarrow \rho^{0} Y} / \mathrm{d} t$ increase with increasing $-t$. Decay-angle analyses for $\rho^{0}, \phi$ and $J / \psi$ mesons have been carried out. For the $\rho^{0}$ and $\phi$ mesons, contributions from single and double helicity flip are observed. The results are compared to expectations of theoretical models.
\end{abstract}




\section{The ZEUS Collaboration}

S. Chekanov, D. Krakauer, S. Magill, B. Musgrave, J. Repond, R. Yoshida

Argonne National Laboratory, Argonne, Illinois 60439-4815 ${ }^{n}$

M.C.K. Mattingly

Andrews University, Berrien Springs, Michigan 49104-0380

P. Antonioli, G. Bari, M. Basile, L. Bellagamba, D. Boscherini, A. Bruni, G. Bruni,

G. Cara Romeo, L. Cifarelli, F. Cindolo, A. Contin, M. Corradi, S. De Pasquale, P. Giusti,

G. Iacobucci, G. Levi, A. Margotti, R. Nania, F. Palmonari, A. Pesci, G. Sartorelli,

A. Zichichi

University and INFN Bologna, Bologna, Italy ${ }^{e}$

G. Aghuzumtsyan, D. Bartsch, I. Brock, J. Crittenden ${ }^{1}$, S. Goers, H. Hartmann, E. Hilger, P. Irrgang, H.-P. Jakob, A. Kappes, U.F. Katz ${ }^{2}$, R. Kerger ${ }^{3}$, O. Kind, E. Paul, J. Rautenberg ${ }^{4}$, R. Renner, H. Schnurbusch, A. Stifutkin, J. Tandler, K.C. Voss, A. Weber

Physikalisches Institut der Universität Bonn, Bonn, Germany ${ }^{b}$

D.S. Bailey ${ }^{5}$, N.H. Brook ${ }^{5}$, J.E. Cole, B. Foster, G.P. Heath, H.F. Heath, S. Robins, E. Rodrigues ${ }^{6}$, J. Scott, R.J. Tapper, M. Wing

H.H. Wills Physics Laboratory, University of Bristol, Bristol, United Kingdom ${ }^{m}$

M. Capua, A. Mastroberardino, M. Schioppa, G. Susinno

Calabria University, Physics Department and INFN, Cosenza, Italy ${ }^{e}$

J.Y. Kim, Y.K. Kim, J.H. Lee, I.T. Lim, M.Y. Pac ${ }^{7}$

Chonnam National University, Kwangju, Korea ${ }^{g}$

A. Caldwell, M. Helbich, X. Liu, B. Mellado, S. Paganis, W.B. Schmidke, F. Sciulli

Nevis Laboratories, Columbia University, Irvington on Hudson, New York 10027o

J. Chwastowski, A. Eskreys, J. Figiel, K. Olkiewicz, K. Piotrzkowski ${ }^{8}$, M.B. Przybycień ${ }^{9}$, P. Stopa, L. Zawiejski

Institute of Nuclear Physics, Cracow, Poland ${ }^{i}$

L. Adamczyk, B. Bednarek, I. Grabowska-Bold, K. Jeleń, D. Kisielewska, A.M. Kowal, M. Kowal, T. Kowalski, B. Mindur, M. Przybycień, E. Rulikowska-Zarębska, L. Suszycki, D. Szuba, J. Szuba ${ }^{10}$

Faculty of Physics and Nuclear Techniques, University of Mining and Metallurgy, Cracow, Poland ${ }^{p}$

A. Kotański ${ }^{11}$, W. Słomiński ${ }^{12}$

Department of Physics, Jagellonian University, Cracow, Poland 
L.A.T. Bauerdick ${ }^{13}$, U. Behrens, K. Borras, V. Chiochia, D. Dannheim, M. Derrick ${ }^{14}$, G. Drews, J. Fourletova, A. Fox-Murphy, U. Fricke, A. Geiser, F. Goebel ${ }^{15}$, P. Göttlicher ${ }^{16}$, O. Gutsche, T. Haas, W. Hain, G.F. Hartner, S. Hillert, U. Kötz, H. Kowalski ${ }^{17}$, H. Labes, D. Lelas, B. Löhr, R. Mankel, M. Martínez ${ }^{13}$, M. Moritz, D. Notz, I.-A. Pellmann, M.C. Petrucci, A. Polini, A. Raval, U. Schneekloth, F. Selonke ${ }^{18}$, B. Surrow ${ }^{19}$, H. Wessoleck, R. Wichmann ${ }^{20}$, G. Wolf, C. Youngman, W. Zeuner

Deutsches Elektronen-Synchrotron DESY, Hamburg, Germany

A. Lopez-Duran Viani ${ }^{21}$, A. Meyer, S. Schlenstedt

DESY Zeuthen, Zeuthen, Germany

G. Barbagli, E. Gallo, C. Genta, P. G. Pelfer

University and INFN, Florence, Italy ${ }^{e}$

A. Bamberger, A. Benen, N. Coppola, H. Raach

Fakultät für Physik der Universität Freiburg i.Br., Freiburg i.Br., Germany ${ }^{b}$

M. Bell, P.J. Bussey, A.T. Doyle, C. Glasman, S. Hanlon, S.W. Lee, A. Lupi, G.J. McCance, D.H. Saxon, I.O. Skillicorn

Department of Physics and Astronomy, University of Glasgow, Glasgow, United Kingdom $^{m}$

I. Gialas

Department of Engineering in Management and Finance, Univ. of Aegean, Greece

B. Bodmann, T. Carli, U. Holm, K. Klimek ${ }^{22}$, N. Krumnack, E. Lohrmann, M. Milite, H. Salehi, S. Stonjek ${ }^{23}$, K. Wick, A. Ziegler, Ar. Ziegler

Hamburg University, Institute of Exp. Physics, Hamburg, Germany ${ }^{b}$

C. Collins-Tooth, C. Foudas, R. Gonçalo ${ }^{6}$, K.R. Long, F. Metlica, D.B. Miller, A.D. Tapper, R. Walker

Imperial College London, High Energy Nuclear Physics Group, London, United Kingdom $^{m}$

P. Cloth, D. Filges

Forschungszentrum Jülich, Institut für Kernphysik, Jülich, Germany

M. Kuze, K. Nagano, K. Tokushuku²4, S. Yamada, Y. Yamazaki

Institute of Particle and Nuclear Studies, KEK, Tsukuba, Japan ${ }^{f}$

A.N. Barakbaev, E.G. Boos, N.S. Pokrovskiy, B.O. Zhautykov

Institute of Physics and Technology of Ministry of Education and Science of Kazakhstan, Almaty, Kazakhstan

H. Lim, D. Son

Kyungpook National University, Taegu, Korea ${ }^{g}$ 
F. Barreiro, O. González, L. Labarga, J. del Peso, I. Redondo ${ }^{25}$, J. Terrón, M. Vázquez Departamento de Física Teórica, Universidad Autónoma Madrid,Madrid, Spain ${ }^{l}$

M. Barbi, A. Bertolin, F. Corriveau, A. Ochs, S. Padhi, D.G. Stairs, M. St-Laurent

Department of Physics, McGill University, Montréal, Québec, Canada H3A 2T8 ${ }^{a}$

T. Tsurugai

Meiji Gakuin University, Faculty of General Education, Yokohama, Japan

A. Antonov, V. Bashkirov ${ }^{26}$, P. Danilov, B.A. Dolgoshein, D. Gladkov, V. Sosnovtsev, S. Suchkov

Moscow Engineering Physics Institute, Moscow, Russia ${ }^{j}$

R.K. Dementiev, P.F. Ermolov, Yu.A. Golubkov, I.I. Katkov, L.A. Khein, I.A. Korzhavina, V.A. Kuzmin, B.B. Levchenko, O.Yu. Lukina, A.S. Proskuryakov, L.M. Shcheglova, N.N. Vlasov, S.A. Zotkin

Moscow State University, Institute of Nuclear Physics, Moscow, Russia ${ }^{k}$

C. Bokel, J. Engelen, S. Grijpink, E. Koffeman, P. Kooijman, E. Maddox, A. Pellegrino, S. Schagen, E. Tassi, H. Tiecke, N. Tuning, J.J. Velthuis, L. Wiggers, E. de Wolf NIKHEF and University of Amsterdam, Amsterdam, Netherlands ${ }^{h}$

N. Brümmer, B. Bylsma, L.S. Durkin, J. Gilmore, C.M. Ginsburg, C.L. Kim, T.Y. Ling Physics Department, Ohio State University, Columbus, Ohio 43210 ${ }^{n}$

S. Boogert, A.M. Cooper-Sarkar, R.C.E. Devenish, J. Ferrando, G. Grzelak, T. Matsushita, M. Rigby, O. Ruske ${ }^{27}$, M.R. Sutton, R. Walczak

Department of Physics, University of Oxford, Oxford United Kingdom ${ }^{m}$

R. Brugnera, R. Carlin, F. Dal Corso, S. Dusini, A. Garfagnini, S. Limentani, A. Longhin, A. Parenti, M. Posocco, L. Stanco, M. Turcato

Dipartimento di Fisica dell' Università and INFN, Padova, Italy ${ }^{e}$

E.A. Heaphy, B.Y. Oh, P.R.B. Saull ${ }^{28}$, J.J. Whitmore ${ }^{29}$

Department of Physics, Pennsylvania State University, University Park, Pennsylvania $16802^{\circ}$

Y. Iga

Polytechnic University, Sagamihara, Japan ${ }^{f}$

G. D’Agostini, G. Marini, A. Nigro

Dipartimento di Fisica, Università 'La Sapienza' and INFN, Rome, Italy ${ }^{e}$

C. Cormack, J.C. Hart, N.A. McCubbin

Rutherford Appleton Laboratory, Chilton, Didcot, Oxon, United Kingdom ${ }^{m}$ 
C. Heusch

University of California, Santa Cruz, California $95064^{n}$

I.H. Park

Seoul National University, Seoul, Korea

N. Pavel

Fachbereich Physik der Universität-Gesamthochschule Siegen, Germany

H. Abramowicz, S. Dagan, A. Gabareen, S. Kananov, A. Kreisel, A. Levy

Raymond and Beverly Sackler Faculty of Exact Sciences, School of Physics, Tel-Aviv University, Tel-Aviv, Israel ${ }^{d}$

T. Abe, T. Fusayasu, T. Kohno, K. Umemori, T. Yamashita

Department of Physics, University of Tokyo, Tokyo, Japan ${ }^{f}$

R. Hamatsu, T. Hirose ${ }^{18}$, M. Inuzuka, S. Kitamura ${ }^{30}$, K. Matsuzawa, T. Nishimura

Tokyo Metropolitan University, Deptartment of Physics, Tokyo, Japan ${ }^{f}$

M. Arneodo ${ }^{31}$, N. Cartiglia, R. Cirio, M. Costa, M.I. Ferrero, S. Maselli, V. Monaco, C. Peroni, M. Ruspa, R. Sacchi, A. Solano, A. Staiano

Università di Torino, Dipartimento di Fisica Sperimentale and INFN, Torino, Italy ${ }^{e}$

R. Galea, T. Koop, G.M. Levman, J.F. Martin, A. Mirea, A. Sabetfakhri

Department of Physics, University of Toronto, Toronto, Ontario, Canada M5S 1A7a

J.M. Butterworth, C. Gwenlan, R. Hall-Wilton, T.W. Jones, J.B. Lane, M.S. Lightwood, J.H. Loizides ${ }^{32}$, B.J. West

Physics and Astronomy Department, University College London, London, United Kingdom $^{m}$

J. Ciborowski ${ }^{33}$, R. Ciesielski ${ }^{34}$, R.J. Nowak, J.M. Pawlak, B. Smalska ${ }^{35}$, J. Sztuk ${ }^{36}$, T. Tymieniecka ${ }^{37}$, A. Ukleja ${ }^{37}$, J. Ukleja, J.A. Zakrzewski, A.F. Żarnecki

Warsaw University, Institute of Experimental Physics, Warsaw, Poland ${ }^{q}$

M. Adamus, P. Plucinski

Institute for Nuclear Studies, Warsaw, Poland ${ }^{q}$

Y. Eisenberg, L.K. Gladilin ${ }^{38}$, D. Hochman, U. Karshon

Department of Particle Physics, Weizmann Institute, Rehovot, Israel ${ }^{c}$

D. Kçira, S. Lammers, L. Li, D.D. Reeder, A.A. Savin, W.H. Smith

Department of Physics, University of Wisconsin, Madison, Wisconsin $53706^{n}$

A. Deshpande, S. Dhawan, V.W. Hughes, P.B. Straub

Department of Physics, Yale University, New Haven, Connecticut 06520-8121 ${ }^{n}$

S. Bhadra, C.D. Catterall, S. Fourletov, S. Menary, M. Soares, J. Standage

Department of Physics, York University, Ontario, Canada M3J 1P3 ${ }^{a}$ 
${ }^{1}$ now at Cornell University, Ithaca/NY, USA

2 on leave of absence at University of Erlangen-Nürnberg, Germany

${ }^{3}$ now at Ministère de la Culture, de L'Enseignement Supérieur et de la Recherche, Luxembourg

${ }^{4}$ supported by the GIF, contract I-523-13.7/97

5 PPARC Advanced fellow

${ }^{6}$ supported by the Portuguese Foundation for Science and Technology (FCT)

${ }^{7}$ now at Dongshin University, Naju, Korea

8 now at Université Catholique de Louvain, Louvain-la-Neuve/Belgium

${ }^{9}$ now at Northwestern Univ., Evanston/IL, USA

${ }^{10}$ partly supported by the Israel Science Foundation and the Israel Ministry of Science

11 supported by the Polish State Committee for Scientific Research, grant no. 2 P03B 09322

12 member of Dept. of Computer Science, supported by the Polish State Committee for Sci. Res., grant no. 2 P03B 06116

13 now at Fermilab, Batavia/IL, USA

14 on leave from Argonne National Laboratory, USA

15 now at Max-Planck-Institut für Physik, München/Germany

16 now at DESY group FEB

17 on leave of absence at Columbia Univ., Nevis Labs., N.Y./USA

18 retired

19 now at Brookhaven National Lab., Upton/NY, USA

${ }^{20}$ now at Mobilcom AG, Rendsburg-Büdelsdorf, Germany

${ }^{21}$ now at Deutsche Börse Systems AG, Frankfurt/Main, Germany

22 supported by the Polish State Committee for Scientific Research, grant no. 5 P03B 08720

23 supported by NIKHEF, Amsterdam/NL

24 also at University of Tokyo

${ }^{25}$ now at LPNHE Ecole Polytechnique, Paris, France

${ }^{26}$ now at Loma Linda University, Loma Linda, CA, USA

27 now at IBM Global Services, Frankfurt/Main, Germany

28 now at National Research Council, Ottawa/Canada

${ }^{29}$ on leave of absence at The National Science Foundation, Arlington, VA/USA

30 present address: Tokyo Metropolitan University of Health Sciences, Tokyo 116-8551, Japan

31 also at Università del Piemonte Orientale, Novara, Italy

32 supported by Argonne National Laboratory, USA

33 also at Eódź University, Poland

34 supported by the Polish State Committee for Scientific Research, grant no. 2 P03B 
07222

${ }^{35}$ supported by the Polish State Committee for Scientific Research, grant no. 2 P03B 00219

${ }^{36}$ Łódź University, Poland

${ }^{37}$ sup. by Pol. State Com. for Scien. Res., 5 P03B 09820 and by Germ. Fed. Min. for Edu. and Research (BMBF), POL 01/043

38 on leave from MSU, partly supported by University of Wisconsin via the U.S.-Israel BSF 
a supported by the Natural Sciences and Engineering Research Council of Canada (NSERC)

$b$ supported by the German Federal Ministry for Education and Research (BMBF), under contract numbers HZ1GUA 2, HZ1GUB 0, HZ1PDA 5, HZ1VFA 5

c supported by the MINERVA Gesellschaft für Forschung GmbH, the Israel Science Foundation, the U.S.-Israel Binational Science Foundation, the Israel Ministry of Science and the Benozyio Center for High Energy Physics

$d$ supported by the German-Israeli Foundation, the Israel Science Foundation, and by the Israel Ministry of Science

$e$ supported by the Italian National Institute for Nuclear Physics (INFN)

$f$ supported by the Japanese Ministry of Education, Science and Culture (the Monbusho) and its grants for Scientific Research

$g$ supported by the Korean Ministry of Education and Korea Science and Engineering Foundation

$h$ supported by the Netherlands Foundation for Research on Matter (FOM)

$i$ supported by the Polish State Committee for Scientific Research, grant no. 620/E-77/SPUB-M/DESY/P-03/DZ 247/2000-2002

$j$ partially supported by the German Federal Ministry for Education and Research (BMBF)

$k$ supported by the Fund for Fundamental Research of Russian Ministry for Science and Education and by the German Federal Ministry for Education and Research (BMBF)

$l$ supported by the Spanish Ministry of Education and Science through funds provided by CICYT

$m$ supported by the Particle Physics and Astronomy Research Council, UK

$n$ supported by the US Department of Energy

$o$ supported by the US National Science Foundation

$p$ supported by the Polish State Committee for Scientific Research, grant no. 112/E-356/SPUB-M/DESY/P-03/DZ 301/2000-2002, 2 P03B 13922

$q$ supported by the Polish State Committee for Scientific Research, grant no. 115/E-343/SPUB-M/DESY/P-03/DZ 121/2001-2002, 2 P03B 07022 


\section{Introduction}

Studies of the elastic production of vector mesons in electron-proton interactions [1] show that at large $Q^{2}$, the exchanged-photon virtuality, or at high vector-meson mass, the cross section increases with energy faster than is observed in hadron-hadron interactions [2,3]. The latter increase is well described by Regge theory [4]. Although the energy dependence of vector-meson production in ep interactions can be described by models based on Regge phenomenology [5] and the vector dominance model (VDM) [6], it can also be explained by models based on perturbative QCD (pQCD) [7,8]. In this case, it is related to the rise of the gluon density in the proton as $x$ decreases, where $x$ is the Bjorken scaling variable. In pQCD models, a perturbative (hard) scale can be provided by either a high $Q^{2}$ or a large meson mass. It is also predicted [9] that, in diffractive dissociation of hadrons, the squared four-momentum transfer, $t$, may serve as a hard scale.

The subject of this paper is vector-meson $(V)$ photoproduction at high $-t$, which is dominated by the proton-dissociative reaction, $\gamma p \rightarrow V Y$, where $Y$ is the dissociated hadronic system. The data cover the photon-proton centre-of-mass energy range $80<W<120$ $\mathrm{GeV}$ and extend from $-t=1.1 \mathrm{GeV}^{2}$ up to $-t=12 \mathrm{GeV}^{2}$ for the $\rho^{0}$, to $7 \mathrm{GeV}^{2}$ for the $\phi$ and to $6.5 \mathrm{GeV}^{2}$ for the $J / \psi$ meson. The vector mesons were identified via their decays to two oppositely charged particles: $\rho^{0} \rightarrow \pi^{+} \pi^{-}, \phi \rightarrow K^{+} K^{-}$, and $J / \psi \rightarrow l^{+} l^{-}\left(e^{+} e^{-}, \mu^{+} \mu^{-}\right)$.

\section{Theoretical approaches}

\subsection{The Regge model}

In Regge theory, diffractive processes are assumed to proceed through the exchange of the Pomeron trajectory. The vector dominance model, schematically indicated in Fig. 四(a), together with Regge theory, gives the following form for the double-differential cross section for the reaction $\gamma p \rightarrow V Y$ :

$$
\frac{\mathrm{d}^{2} \sigma_{\gamma p \rightarrow V Y}}{\mathrm{~d} M_{Y}^{2} \mathrm{~d} t}=f(t) \frac{1}{W^{2}}\left(\frac{W^{2}}{M_{Y}^{2}}\right)^{2 \alpha(t)-1}\left(M_{Y}^{2}\right)^{\alpha(0)-1},
$$

where $M_{Y}$ is the mass of the diffractively produced hadronic state, $Y$.

Assuming a linear form for the Pomeron trajectory, $\alpha(t)=\alpha(0)+\alpha^{\prime} t$, fits $[10,11]$ to the elastic photoproduction of $\rho^{0}, \phi$ and $J / \psi$ mesons in the range $-t<1.5 \mathrm{GeV}^{2}$ gave $\alpha(0)=1.096 \pm 0.021$ and $\alpha^{\prime}=0.125 \pm 0.038 \mathrm{GeV}^{-2}$ for the $\rho^{0}, \alpha(0)=1.081 \pm 0.010$ and $\alpha^{\prime}=0.158 \pm 0.028 \mathrm{GeV}^{-2}$ for the $\phi$, and $\alpha(0)=1.200 \pm 0.009_{-0.010}^{+0.004}$ and $\alpha^{\prime}=0.115 \pm$ $0.018_{-0.015}^{+0.008} \mathrm{GeV}^{-2}$ for the $J / \psi$ meson. These values may be compared to $\alpha(0)=1.08[2]$, 
$\alpha(0)=1.096$ [3] and $\alpha^{\prime}=0.25 \mathrm{GeV}^{-2}$ [2], obtained from fits to hadron-scattering data at $-t<0.5 \mathrm{GeV}^{2}$. These numbers indicate that the Pomeron trajectory is not universal. In fact, it has been suggested [12] that, in the production of vector mesons, an anomalous Regge trajectory exchange, with $\alpha(0) \approx 1$ and $\alpha^{\prime} \approx 0$, gives a dominant contribution to the differential cross section at large $-t$.

\section{2 pQCD models}

In models based on pQCD [13-18], the reaction $\gamma p \rightarrow V Y$ is viewed as a sequence of three successive processes, illustrated in Figs. 1 (b) and 目(c): the photon fluctuates into a $q \bar{q}$ pair; the $q \bar{q}$ pair scatters off a single parton in the proton by the exchange of a colour singlet; the scattered $q \bar{q}$ pair becomes a vector meson and the struck parton and the proton remnant fragment into a system of hadrons. The probability of the photon fluctuating into a $q \bar{q}$ pair is parameterised by the photon wave-function. Many models assume that the interaction of the $q \bar{q}$ pair with a parton in the proton is mediated in the lowest order by the exchange of two gluons $[13,15,17,18]$. The exchange of the gluon ladder has also been computed $[13,14,16,18]$ in the leading logarithm approximation (LLA). The transition of a $q \bar{q}$ pair into a meson is, however, a non-perturbative phenomenon that must be parameterised by the meson wave-function derived from lattice calculations and sum rules.

In the perturbative regime, the cross section for the process $\gamma p \rightarrow V Y$ is given by

$$
\frac{\mathrm{d}^{2} \sigma_{\gamma p \rightarrow V Y}}{\mathrm{~d} t \mathrm{~d} x}=g(x,-t) \frac{\mathrm{d} \sigma_{\gamma g \rightarrow V g}}{\mathrm{~d} t}+\sum(q(x,-t)+\bar{q}(x,-t)) \frac{\mathrm{d} \sigma_{\gamma q \rightarrow V q}}{\mathrm{~d} t}
$$

where $x$ is the fraction of the proton momentum carried by the struck parton and $g(x,-t)$, $q(x,-t)$ and $\bar{q}(x,-t)$ are the gluon, quark and antiquark density functions, respectively. The cross section for the production of a vector meson through the interaction with a gluon (Fig. 目(c)) is about five times larger than through the interaction with a quark (Fig. [1(b)). At low $x$, therefore, the process $\gamma p \rightarrow V Y$ should be dominated by the reaction in which a gluon from the proton is struck.

In the two-gluon approximation, the cross section of Eq. (2) does not depend on $W$ for any given $t$, i.e. $\alpha^{\prime}=0$. In the LLA, this cross section has a power-law dependence on $W$, although $\alpha^{\prime}$ is expected to be small, $\alpha^{\prime} \lesssim 0.1 \mathrm{GeV}^{-2}$ for $-t \gtrsim 1 \mathrm{GeV}^{2}$ [16], decreasing with increasing $-t$ and reaching zero at asymptotically large $-t$ values.

A previous ZEUS publication [10] showed that $\mathrm{d} \sigma / \mathrm{d} t$ for the $\gamma p \rightarrow \rho^{0} Y$ and $\gamma p \rightarrow \phi Y$ processes were larger than those obtained by perturbative calculations performed in the two-gluon approximation [15]. These calculations have been further developed by Ivanov et al. [17], who proposed that a large contribution would arise from $q \bar{q}$ fluctuations in a 
chiral-odd spin configuration. In this case, the quark mass appearing in the $\gamma V$ coupling is interpreted as a current quark mass, and therefore the quark mass in the light-meson wave-function is neglected. In such an approximation, $\mathrm{d} \sigma / \mathrm{d} t$ for $\rho^{0}$ and $\phi$ mesons should have the same shape for $-t \gtrsim 1 \mathrm{GeV}^{2}$. In addition, the cross sections should exhibit a power-law dependence on $t, \mathrm{~d} \sigma / \mathrm{d} t \propto(-t)^{-n}$; for $x>0.01$, the exponent $n \approx 3.8$ can be estimated from the Ivanov et al. calculation [17] for a fixed value of the strong coupling constant, $\alpha_{s}$, or $n \approx 4.8$ using the leading-order form for $\alpha_{s}(-t)$ [19].

In another two-gluon calculation, that of Forshaw and Ryskin [13], the quark mass appearing in the $\gamma V$ coupling is interpreted as the constituent quark mass. Such models predict a dip in $\mathrm{d} \sigma / \mathrm{d} t$ at $-t \approx M_{V}^{2}$, where $M_{V}$ is the meson mass.

A LLA BFKL [20] calculation has been carried out by Bartels et al. [16] for heavy mesons. The results are expanded in terms of $\ln \left(x W^{2} / W_{0}^{2}\right)$, where $W_{0}^{2}=M_{V}^{2}-t$ is assumed. This model predicts that $\mathrm{d} \sigma / \mathrm{d} t \propto(-t)^{-n}$, where $n$ is a function of the ratio $-t / M_{V}^{2}$. For the $t$ range covered by the $J / \psi$ analysis of this paper, an average value of $n \approx 1.8$ is predicted for a fixed value of $\alpha_{s}$. The previous ZEUS measurement of the $\gamma p \rightarrow J / \psi Y$ cross section [10] shows that the prediction of this model, using $\alpha_{s}=0.2$, agrees well with the data. However, the uncertainties due to the choice of $\alpha_{s}$ and the $W_{0}$ scale are large.

In a recent paper, Forshaw and Poludniowski [18] have extended the BFKL models of Forshaw and Ryskin [13] and Bartels et al. [16], and have given cross sections for the $\rho^{0}$, $\phi$ and $J / \psi$ mesons. In this model, which uses a fixed value for $\alpha_{s}$, a delta-function meson wave-function was used and the quark mass appearing in the $\gamma V$ coupling was assumed to be the constituent quark mass for all three mesons.

\subsection{Helicity structure and cross-section ratios}

The hypothesis of $s$-channel helicity conservation (SCHC) [21] for vector-meson production states that the helicity of the final-state meson will be equal to that of the initial photon. Studies of the elastic photoproduction of light vector mesons at low $-t[1]$ show that SCHC holds in these soft diffractive processes.

In VDM, the cross section for vector-meson production is proportional to the product of the coupling of the photon to the vector meson and the meson-proton scattering cross section. The $\gamma \rightarrow V$ coupling depends on the meson leptonic width, $\Gamma_{V \rightarrow e^{+} e^{-}}$, which depends on the meson mass, the quark assignment and the wave-function. The SU(4) prediction, which ignores the differences in the masses and the wave-functions of the mesons, is that the ratio of the production cross sections for vector mesons will be

$$
\rho^{0}: \omega: \phi: J / \psi=1: 1 / 9: 2 / 9: 8 / 9
$$


In pQCD, the helicity of the final-state meson and the cross-section ratios are sensitive to the photon polarisation and the meson wave-function. In the trivial case of a meson wave-function given by a delta function, which is a good approximation for heavy vector mesons, the helicity of the final-state vector meson is equal to that of the initial photon, and thus SCHC will hold. In this case, the cross-section for transverse photons is given by $\mathrm{d} \sigma / \mathrm{d} t \propto M_{V}^{3} \Gamma_{V \rightarrow e^{+} e^{-}}[13,16,18]$. Based on the measured values of $M_{V}$ and $\Gamma_{V \rightarrow e^{+} e^{-}}[22]$, the ratio of the photoproduction cross sections for various vector mesons at asymptotic values of $-t$ becomes

$$
\rho^{0}: \omega: \phi: J / \psi=1:(0.8 \times 1 / 9):(2.1 \times 2 / 9):(56 \times 8 / 9),
$$

which is significantly higher than the $\mathrm{SU}(4)$ prediction of Eq. (3) for the $\phi$ and $J / \psi$ meson.

A wave-function more appropriate for the light mesons $[15,17]$ leads to the production of mesons in the helicity- 0 state by transverse photons and therefore to a violation of $\mathrm{SCHC}$. The ratios of the production cross sections for mesons in the helicity- \pm 1 states by transverse photons are equal to those of Eq. (4). The production of mesons in the helicity-0 state by transverse photons, which is expected to be dominant at large $-t$, is given by $\mathrm{d} \sigma / \mathrm{d} t \propto M_{V} \Gamma_{V \rightarrow e^{+} e^{-}}$rather than $M_{V}^{3} \Gamma_{V \rightarrow e^{+} e^{-}}$mentioned earlier. In this case, the ratios of the photoproduction cross sections at large $-t$ should be

$$
\rho^{0}: \omega: \phi: J / \psi=1:(0.8 \times 1 / 9):(1.2 \times 2 / 9):(3.5 \times 8 / 9),
$$

which lie between the values of Eqs. (3) and (44).

\section{Experimental set-up}

The measurements were performed with the ZEUS detector at the HERA ep collider. The data, corresponding to an integrated luminosity of $25.0 \pm 0.4 \mathrm{pb}^{-1}$, were collected in 1996 and 1997, when HERA operated with a proton-beam energy of $820 \mathrm{GeV}$ and a positron-beam energy of $27.5 \mathrm{GeV}$.

A detailed description of the ZEUS detector can be found elsewhere [23]. A brief outline of the components that are most relevant for this analysis is given below.

Charged particles are tracked in the central tracking detector (CTD) [24], which operates in a magnetic field of $1.43 \mathrm{~T}$ provided by a thin superconducting coil. The CTD consists of 72 cylindrical drift chamber layers, organized in 9 superlayers covering the 
polar-angle円 region $15^{\circ}<\theta<164^{\circ}$. The transverse-momentum resolution for full-length tracks is $\sigma\left(p_{T}\right) / p_{T}=0.0058 p_{T} \oplus 0.0065 \oplus 0.0014 / p_{T}$, with $p_{T}$ in $\mathrm{GeV}$.

The high-resolution uranium-scintillator calorimeter (CAL) [25] consists of three parts: the forward (FCAL), the barrel (BCAL) and the rear (RCAL) calorimeters. Each part is subdivided transversely into towers and longitudinally into one electromagnetic section (EMC) and either one (in RCAL) or two (in BCAL and FCAL) hadronic sections (HAC). The smallest subdivision of the calorimeter is called a cell. The CAL energy resolutions, as measured under test-beam conditions, are $\sigma(E) / E=0.18 / \sqrt{E}$ for electrons and $\sigma(E) / E=0.35 / \sqrt{E}$ for hadrons $(E$ in $\mathrm{GeV})$.

A proton-remnant tagger (PRT1) [26] was used to tag events in which the proton dissociates. It consists of two layers of scintillation counters at $Z=5.15 \mathrm{~m}$. The pseudorapidity range covered by the PRT1 is $4.3<\eta<5.8$.

The photoproduction tagger (PT) [10] is a small electromagnetic calorimeter located close to the beam-pipe at $Z=-44 \mathrm{~m}$. It detects positrons of energy between 21 and $26 \mathrm{GeV}$ scattered under very small angles (less than a few mrads). The positron measured in the PT is used to tag photoproduction events with a photon-beam energy in the $1.5-6.5 \mathrm{GeV}$ range.

The luminosity is determined from the rate of the Bethe-Heitler bremsstrahlung process $e^{+} p \rightarrow e^{+} \gamma p$, where the high-energy photon is detected in a lead-scintillator calorimeter (LUMI) located at $Z=-107 \mathrm{~m}$ in the HERA tunnel [27].

\section{Kinematics and decay distributions}

Figure 1(d) shows a diagram of the reaction

$$
e(k) p(P) \rightarrow e\left(k^{\prime}\right) V(v) Y\left(P^{\prime}\right)
$$

where $V$ is a $\rho^{0}, \phi$, or $J / \psi$ meson and $k, k^{\prime}, P, P^{\prime}$ and $v$ are the four-momenta of the incident positron, scattered positron, incident proton, diffractively produced state $Y$ and vector meson, respectively. The kinematic variables used to describe proton-dissociative vector-meson production are:

\footnotetext{
${ }^{1}$ The ZEUS coordinate system is a right-handed Cartesian system, with the $Z$ axis pointing in the proton beam direction, referred to as the "forward direction", and the $X$ axis pointing left towards the centre of HERA. The coordinate origin is at the nominal interaction point. The pseudorapidity is defined as $\eta=-\ln \left(\tan \frac{\theta}{2}\right)$, where the polar angle, $\theta$, is measured with respect to the proton beam direction.
} 
- $Q^{2}=-q^{2}=-\left(k-k^{\prime}\right)^{2}$, the negative of the squared four-momentum of the exchanged photon;

- $y=(P \cdot q) /(P \cdot k)$, the fraction of the positron energy transferred to the photon in the rest frame of the initial-state proton;

- $W^{2}=(q+P)^{2}$, the squared centre-of-mass energy of the photon-proton system;

- $t=\left(P-P^{\prime}\right)^{2}=(v-q)^{2}$, the squared four-momentum exchanged at the hadronic vertex;

- $x$, the fraction of the proton's momentum carried by the scattered parton. Assuming that the exchanged object in the photon-proton scattering couples to a single massless parton in the proton

$$
x=\frac{-\left(P^{\prime}-P\right)^{2}}{2 P\left(P^{\prime}-P\right)}=-t /\left(M_{Y}^{2}-M_{p}^{2}-t\right),
$$

where $M_{p}$ is the mass of the proton and $M_{Y}^{2}=\left(P^{\prime}\right)^{2}$ is the squared mass of the diffractively produced state, $Y$.

The angles used are shown in Fig. 2 and are:

- $\Phi$, the angle, in the photon-proton centre-of-mass frame, between the positron-scattering plane and the $V$-production plane. The latter contains the momentum vectors of the virtual photon and the vector meson;

- $\theta_{h}$ and $\varphi_{h}$, the polar and azimuthal angles of the positively charged decay particle in the $V$ helicity frame. The helicity frame is the rest frame of the meson with the meson direction in the photon-proton centre-of-mass frame taken as the quantisation axis. The polar angle, $\theta_{h}$, is defined as the angle between the direction of the positively charged decay particle and the quantisation axis. The azimuthal angle, $\varphi_{h}$, is the angle between the decay plane and the $V$-production plane.

Only the momentum vectors of the meson decay products were measured. The momentum of the scattered positron (and hence $Q^{2}$ ), the angle $\Phi$, and the mass $M_{Y}$ were not measured.

In the tagged photoproduction events, $Q^{2}$ ranges from $Q_{\min }^{2} \approx 10^{-9} \mathrm{GeV}^{2}$ up to $Q_{\max }^{2} \approx$ $0.02 \mathrm{GeV}^{2}$, with a median $Q^{2}$ of approximately $7 \times 10^{-6} \mathrm{GeV}^{2}[10]$. The value of $Q^{2}$ was neglected in the reconstruction of the other kinematic variables.

The variables $W$ and $t$ can be expressed as [10]

$$
W^{2} \approx 2 E_{p}\left(E-p_{Z}\right) \text { and } t \approx-p_{T}^{2}
$$


where $E_{p}$ is the incoming proton energy and $E$ is the energy, $p_{Z}$ is the longitudinal momentum and $p_{T}$ is the transverse momentum of the produced vector meson in the laboratory frame.

Since $\Phi$ is not measured, the three-dimensional angular distribution was averaged over this angle, so that the \pm 1 photon-helicity states are not distinguished. The normalised two-dimensional angular distribution can then be written in terms of spin density matrix elements as

$$
\begin{aligned}
\frac{1}{\sigma} \frac{\mathrm{d}^{2} \sigma}{\mathrm{d} \cos \theta_{h} \mathrm{~d} \varphi_{h}}=\frac{3}{4 \pi} & \left\{\frac{1}{2}\left(1 \mp r_{00}^{04}\right) \pm \frac{1}{2}\left(3 r_{00}^{04}-1\right) \cos ^{2} \theta_{h}\right. \\
& \left.\mp \sqrt{2} \operatorname{Re}\left[r_{10}^{04}\right] \sin 2 \theta_{h} \cos \varphi_{h} \mp r_{1-1}^{04} \sin ^{2} \theta_{h} \cos 2 \varphi_{h}\right\},
\end{aligned}
$$

where the upper (lower) signs are for the $V$ decay to spin-0 (spin-1/2) particles. Integration over $\theta_{h}$ or $\varphi_{h}$ produces the one-dimensional distributions

$$
\frac{\mathrm{d} \sigma}{\mathrm{d} \cos \theta_{h}} \propto 1 \mp r_{00}^{04} \pm\left(3 r_{00}^{04}-1\right) \cos ^{2} \theta_{h}
$$

and

$$
\frac{\mathrm{d} \sigma}{\mathrm{d} \varphi_{h}} \propto 1 \mp r_{1-1}^{04} \cos 2 \varphi_{h}
$$

Since $Q^{2}$ is very small for this study, the contribution from photons in the helicity-0 state is negligible. In this case, the matrix elements are related to the helicity amplitudes, $T_{\lambda_{V} \lambda_{\gamma}}$, as follows [28]:

$$
r_{00}^{04} \approx \frac{T_{01}^{2}}{T_{01}^{2}+T_{11}^{2}+T_{-11}^{2}}, r_{10}^{04} \approx \frac{1}{2} \frac{\left(T_{11} T_{01}^{\star}\right)+\left(T_{-11} T_{01}^{\star}\right)}{T_{01}^{2}+T_{11}^{2}+T_{-11}^{2}}, r_{1-1}^{04} \approx \frac{1}{2} \frac{\left(T_{11} T_{-11}^{\star}\right)+\left(T_{-11} T_{11}^{\star}\right)}{T_{01}^{2}+T_{11}^{2}+T_{-11}^{2}},
$$

where $T_{11}$ is the helicity non-flip amplitude, $T_{01}$ is the helicity single-flip amplitude and $T_{-11}$ is the helicity double-flip amplitude. If SCHC holds, only the $T_{11}$ amplitude is non-zero and therefore $r_{00}^{04}, r_{10}^{04}$ and $r_{1-1}^{04}$ should all be zero.

\section{Event selection}

Events were selected online with a three-level trigger system [23]. The selection of the reaction $e p \rightarrow e V Y$ at large values of $-t$ required:

- at the first trigger level:

- a minimum energy deposit of $1 \mathrm{GeV}$ in the PT; 
- at least one track candidate in the CTD.

In addition, the following cuts were applied to further reduce the backgrounds from random coincidences between some activity in the CTD and a detected positron in the PT:

- an upper limit of $1 \mathrm{GeV}$ on the energy deposited in the LUMI photon detector. This requirement suppresses bremsstrahlung events and rejects hard QED radiation;

- at least $0.5 \mathrm{GeV}$ of energy deposit in the EMC section of the RCAL. This cut rejects proton-gas events. However, it also reduces the acceptance for $e p \rightarrow e V Y$ events and therefore was used only when necessary;

- at the second trigger level:

- CAL timing consistent with an ep collision;

- not more than three tracks in the CTD;

- at the third trigger level:

- exactly two tracks in the CTD pointing to a common vertex within $-60<Z<$ $60 \mathrm{~cm}$;

- the transverse momentum of at least one track candidate greater than $0.8 \mathrm{GeV}$. This cut efficiently selects events with large values of $-t$.

After the event reconstruction, the following requirements were imposed:

- an interaction vertex within $-40<Z<40 \mathrm{~cm}$ and a transverse distance within $0.7 \mathrm{~cm}$ of the nominal interaction point (IP);

- exactly two tracks with opposite charges, each associated with the reconstructed event vertex, and each with pseudorapidity $|\eta|<2.1$ and transverse momentum greater than $150 \mathrm{MeV}$ for the $\rho^{0}, 400 \mathrm{MeV}$ for the $\phi$, and $500 \mathrm{MeV}$ for the $J / \psi$ candidates;

- energy deposits in the CAL (excluding FCAL cells with $\eta>2.1$ ), not associated with tracks, smaller than $250 \mathrm{MeV}$. This cut rejects events with an additional particle not associated with either the vector meson or the proton-dissociative system.

In addition, $-t>1.1 \mathrm{GeV}^{2}$ and $80<W<120 \mathrm{GeV}$ were required to select a kinematic region of high acceptance. The final number of events, after all selection cuts, was 21414 for $0.55<M_{\pi \pi}<1.3 \mathrm{GeV}, 2407$ for $0.99<M_{K K}<1.06 \mathrm{GeV}$ and 214 for $2.7<M_{l l}<$ $3.2 \mathrm{GeV}$.

\section{Monte Carlo simulation and acceptance calculation}




\subsection{Monte Carlo simulation}

The EPSOFT [29] Monte Carlo (MC) generator was used for the simulation of the reaction $\gamma p \rightarrow V Y$. The particular version [30] used here had an improved simulation of the finalstate particle multiplicity. The DIFFVM [31] MC generator was used for systematic checks. In these generators, $\gamma p$ interactions are simulated assuming the exchange of a colourless object. The cross-section $\mathrm{d}^{2} \sigma_{\gamma p \rightarrow V Y} /\left(\mathrm{d} t \mathrm{~d} M_{Y}^{2}\right)$ was parameterised using an exponential $t$ distribution and a $1 / M_{Y}^{2}$ distribution; SCHC was also assumed. Because the MC samples thus generated did not give a sufficiently good description of the data, they were reweighted as described in the next section. The only difference between the EPSOFT and DIFFVM generators that is relevant for this analysis is the fragmentation scheme of system $Y$ :

- in EPSOFT, the exchanged object is assumed to couple to the whole proton (Fig. 1 (a)), which subsequently fragments into a state $Y$. The particle multiplicities and the transverse momenta of the hadrons in $Y$ are simulated using parameterisations of $p p$ data [32], while the longitudinal momenta are generated with a uniform rapidity distribution;

- in DIFFVM, the exchanged object couples to a single quark (Fig. 1(b)) in the proton. The fraction of the proton's momentum carried by the struck quark is given by Eq. (6). The struck quark and the diquark remnant are colour-connected and are fragmented into the final-state $Y$ by the JETSET7.3 [33] program.

As pointed out in Section 2.2, pQCD models predict that the process $\gamma p \rightarrow V Y$ is dominated by the reaction involving a gluon (Fig. 1(c)) from the proton. This process was implemented as an option in DIFFVM. In the following sections, the DIFFVM sample where this option was selected is called DIFFVMg, while the DIFFVM sample in which the proton emits a quark is called DIFFVMq.

The response of the ZEUS detector to generated particles was simulated using a program based on GEANT3.13 [34]. The generated events were processed through the same selection and reconstruction procedures as the data.

\subsection{Modelling of the dissociative-system $Y$}

The FCAL energy distributions are sensitive to the fragmentation schemes of the system $Y$ as well as to the shape of the generated $M_{Y}$ distribution. The latter was reweighted with a function $\left(1 / M_{Y}^{2}\right)^{\beta(t)-1}$ to have the effective dependence

$$
\frac{\mathrm{d} \sigma_{\gamma p \rightarrow V Y}}{\mathrm{~d} M_{Y}^{2}} \propto\left(\frac{1}{M_{Y}^{2}}\right)^{\beta(t)} .
$$


The exponent $\beta(t)$ was chosen so as to reproduce the measured energy distributions in the FCAL for the $\rho^{0}$ data sample: $\beta(t)=0.93 e^{0.11 t}$ for EPSOFT and $\beta(t)=0.67 e^{-0.1 t}$ for DIFFVMg. These parameterisations are also valid for the $\phi$ and $J / \psi$ mesons. The comparisons between the data and the MC simulations are displayed in Fig. 3 for several $t$ ranges and for the three mesons. The agreement is satisfactory for DIFFVMg and EPSOFT but not for DIFFVMq (shown only for the $\rho^{0}$ in Fig. 3). This observation is consistent with the pQCD expectation that the photoproduction of vector mesons with proton dissociation at low $x$ is dominated by the reaction in which a gluon from the proton couples to the vector meson. The DIFFVMg samples were used for systematic checks since the results do not change significantly when the mixture of DIFFVMq and DIFFVMg expected in Eq. (2) was used.

\subsection{Acceptance corrections}

The overall acceptance is the product of the PT acceptance and that of the main detector. The geometric acceptance of the PT was evaluated with a program that uses the HERA beam-transport matrices to track the positron through the HERA beamline. The simulation was tuned so as to reproduce the tagging efficiency of the PT for Bethe-Heitler events, $e p \rightarrow e \gamma p$, triggered by a photon measured in the LUMI photon detector. This procedure was described previously [10]. The variations of the position and tilt of the positron beam at the IP observed during data taking [35] were also simulated.

The efficiency for photoproduction events was determined using the geometric acceptance of the PT and events were generated according to the equivalent-photon approximation [36]. The photoproduction tagging efficiency, calculated as a function of the positron energy, $E_{e^{\prime}}$, is shown in Fig. 1 . The range $80<W<120 \mathrm{GeV}$ was chosen so that the systematic uncertainty of the tagging efficiency, shown by the shaded area, does not dominate the total systematic uncertainty. For the kinematic range used in this analysis, the cross-section-weighted PT acceptance averages $80 \pm 6 \%$ D.

The decay-angle distributions at the generator level were iteratively reweighted according to Eq. (7) using the spin density matrix elements found in this study (see Section 9.6) The spin density matrix elements for the $\rho^{0}$ and $\phi$ mesons were assumed to be linear functions of $t$; SCHC was assumed for the $J / \psi$ meson. The generated $t$ distributions were reweighted with a function $e^{P(t)}$, where $P(t)$ is a polynomial, to reproduce those observed in the data. The resulting simulation agrees reasonably well with the data, as shown in Fig. 可.

\footnotetext{
${ }^{2}$ For these data, the PT was shifted closer to the positron beam, yielding a higher tagging efficiency than in the previous ZEUS measurement [10].
} 
Acceptance corrections evaluated on the basis of the reweighted EPSOFT sample, which gives the best description of the data, were calculated separately for each bin of any given variable.

On average, $6 \%$ of $e p \rightarrow e V Y$ events were rejected by the trigger due to accidental coincidence with bremsstrahlung photons in the LUMI calorimeter. This effect was corrected for in the analysis.

\section{$7 \quad$ Backgrounds}

The dominant background sources, common to all channels, came from the doubledissociative, $\gamma p \rightarrow X Y$, and non-diffractive, $\gamma p \rightarrow$ anything, processes. Additional backgrounds contributing to individual channels are non-resonant $\pi^{+} \pi^{-}$production for the $\rho^{0}$ sample, $\rho^{0}$ production for the $\phi$ sample, and inelastic Bethe-Heitler production of $e^{+} e^{-}$ and $\mu^{+} \mu^{-}$pairs for the $J / \psi$ sample. These backgrounds were statistically subtracted using the fits to the invariant-mass distributions described in Section 9.2 .

The contribution of elastic vector-meson production $(\gamma p \rightarrow V p)$ to the data was estimated from the fraction of events, $F$, with a signal in one of the PRT1 counters above a threshold corresponding to the signal of a minimum-ionising particle. For elastic vector-meson production, such energy deposits are absent. A comparison of the EPSOFT prediction with the measured values of $F$ shows that the contribution from the elastic process decreases from about 0.2 at $-t=1.2 \mathrm{GeV}^{2}$ to about 0.05 at $-t=3.0 \mathrm{GeV}^{2}$. The precision with which this background is known is 0.01 for the $\rho^{0}, 0.03$ for the $\phi$ and 0.1 for the $J / \psi$

meson. This background was statistically subtracted bin by bin. For $-t>3 \mathrm{GeV}^{2}$, the contamination from the elastic reaction was consistent with zero and was neglected.

The contributions from the $\omega$ and $\phi$ mesons (decaying to $\pi^{+} \pi^{-} \pi^{0}$ ) in the $\rho^{0}$-candidate sample were estimated using a MC simulation and the cross-section ratio given by the $\mathrm{SU}(4)$ prediction of $1 / 9$ for $\omega / \rho^{0}$ and the results of this analysis for $\phi / \rho^{0}$ (see Section 9.4). This background was less than $3 \%$. The contribution from the $\psi^{\prime}$ meson (decaying to $J / \psi+$ neutrals) in the $J / \psi$ candidate sample was less than 3\%. These backgrounds contribute to the overall normalisation uncertainty.

\section{Systematic uncertainties}

The overall systematic uncertainties in the cross sections and density matrix elements in each bin were obtained by summing in quadrature the uncertainties listed below:

- event selection: 
- the selection cuts, described in Section 5, were varied within the resolutions of the cut variables;

- a different procedure to associate CTD tracks and CAL objects was used.

The effect on the cross sections was less than $\pm 10 \%$, and less than \pm 0.03 in the spin density matrix elements;

- signal extraction:

- the parameterisations of the signal shape were changed from the functional forms to the MC expectations;

- the ranges of the $M_{\pi \pi}, M_{K K}$ and $M_{l l}$ mass distributions used in the fit procedure described in Section 9.2 were varied by their resolutions.

This resulted in changes of less than $\pm 10 \%$ in the cross sections and less than \pm 0.01 in the spin density matrix elements;

- modelling of the dissociative-system $Y$ :

- $\beta(t)$ in Eq. (11) was changed to $\beta(t)=(0.93 \pm 0.07) e^{(0.11 \pm 0.06) t}$ for the $\rho^{0}, \beta(t)=$ $(0.93 \pm 0.17) e^{(0.19 \pm 0.17) t}$ for the $\phi$ and $\beta(t)=0.73 \pm 0.37$ for the $J / \psi$ meson. These ranges were chosen to maintain a satisfactory agreement between the measured FCAL energy distributions and the EPSOFT simulation;

- the fragmentation scheme of the system $Y$ was changed by using DIFFVMg instead of EPSOFT for the acceptance corrections;

- $M_{Y}^{2}-M_{p}^{2}-t$ was used instead of $M_{Y}^{2}$ [37] in Eq. (11);

- the $1 / M_{Y}^{2}$ dependence was reweighted to agree with the pQCD prediction of Eq. (2) ?.

The resulting uncertainty in the cross sections, coming mainly from the $\beta(t)$ variation, was less than $\pm 20 \%$. All checks in this class change the generated $M_{Y}$ distribution. Therefore, to avoid double counting, the maximum deviation from the nominal value was taken as the total systematic uncertainty of this class of systematic check. Since the MC simulation assumes factorisation of the proton and meson vertices, these systematic checks result in no changes to the spin density matrix elements;

- reweighting of the other MC distributions in EPSOFT:

- the $\theta_{h}$ and $\varphi_{h}$ distributions were reweighted according to the SCHC predictions for the $\rho^{0}$ and $\phi$ mesons. For the $J / \psi$, the values $r_{00}^{04}=0.13$ or $r_{1-1}^{04}= \pm 0.2$ were used, corresponding to one standard deviation from the $\mathrm{SCHC}$ values, as discussed in Section 9.6;

\footnotetext{
3 The GRV98 [19] parton densities were used, but the results do not change when other parameterisations are selected.
} 
- the $t$ and $W$ distributions were reweighted by a power-law function of $t$ and $W$, respectively, whilst maintaining satisfactory agreement between the data and the MC distributions.

These modifications to the analysis procedure lead to changes in the cross section of less than $7 \%$ and less than \pm 0.01 in the spin density matrix elements;

- calorimeter trigger efficiency:

- the EMC RCAL trigger efficiency was varied within its uncertainty, as determined from subsamples of events triggered without the requirement of an energy deposit in the EMC section of the RCAL.

This resulted in changes to the cross sections of less than $\pm 5 \%$ and variations in the spin density matrix elements of up to \pm 0.01 ;

- normalisation uncertainty:

- PT acceptance; the systematic uncertainty related to the PT acceptance was evaluated by varying the inputs to the MC simulation. The energy scale for photons measured in the LUMI detector was changed by $\pm 50 \mathrm{MeV}$ [27], the horizontal position of the PT by $\pm 0.3 \mathrm{~mm}$, and the average position of the IP by $\pm 0.1 \mathrm{~mm}$. These variations correspond to the systematic uncertainties, as calculated from the Bethe-Heitler event sample, discussed in Section 6.3. The effect on the cross sections was $\pm 6 \%$;

- tracking-trigger efficiency; the uncertainty on the CTD trigger efficiency was estimated from an independently triggered data sample and corresponds to an uncertainty on the cross section of $\pm 4 \%$;

- PRT1 acceptance; the systematic uncertainty related to the PRT1 acceptance was estimated from the difference between the fraction of events with a signal in the PRT1 in the data and in the EPSOFT simulation in the region of large $-t$, where the elastic contribution is expected to be negligible. This resulted in a systematic uncertainty of $\pm 3 \%$ in the cross sections. This value was also used in the low $-t$ region;

- other uncertainties; backgrounds from the $\omega$ and $\phi$ mesons in the $\rho^{0}$ sample and from the $\psi^{\prime}$ meson in the $J / \psi$ sample $(-3 \%)$, as discussed in Section 0 ; QED radiation $( \pm 2 \%)$ [10]; measurement of the integrated luminosity $( \pm 1.8 \%)[27]$;

The overall normalisation uncertainty of $\pm 10 \%$ was the sum in quadrature of the above uncertainties. This uncertainty does not affect the spin density matrix elements. 


\section{Results}

Although $M_{Y}$ (and hence $x$ ) was not measured, the trigger and offline selection restricted $M_{Y}$ to be $\lesssim 25 \mathrm{GeV}$, which corresponds to $0.005 \lesssim x<1$ at $-t=2 \mathrm{GeV}^{2}$ and $0.03 \lesssim x<1$ at $-t=10 \mathrm{GeV}^{2}$. To facilitate the comparison of the data with the pQCD predictions, the cross sections presented in the following sections were evaluated in the fixed $x$ range $0.01<x<1$, which approximately corresponds to the $x$ range covered by the data at the average $t$ of this analysis.

\subsection{Cross-section determination}

The differential cross-section $\mathrm{d} \sigma_{\gamma p \rightarrow V Y} / \mathrm{d} \xi$ for the photoproduction process $\gamma p \rightarrow V Y$ was obtained from the cross section measured for the reaction $e p \rightarrow e V Y$ in each bin of the variable $\xi\left(=t, \varphi, \cos \theta_{h}\right)$ as

$$
\frac{\mathrm{d} \sigma_{\gamma p \rightarrow V Y}}{\mathrm{~d} \xi}=\frac{\mathrm{d} \sigma_{e p \rightarrow e V Y}}{\mathrm{~d} \xi} \frac{1}{\Phi_{\gamma}}=\frac{N_{V}}{\Delta \xi \cdot \mathcal{L} \cdot \Phi_{\gamma} \cdot B_{V}},
$$

where $\Phi_{\gamma}$ is the effective photon flux [36], $N_{V}$ is the background-corrected and acceptancecorrected number of vector mesons in the range $\Delta \xi$, extracted using the fits described in Section 9.2, $\mathcal{L}$ is the integrated luminosity and $B_{V}$ is the branching ratio of the vectormeson decay channel. The cross sections for $\rho^{0}$ production were integrated over the range $2 M_{\pi}<M_{\pi \pi}<M_{\rho}+5 \Gamma_{\rho}$.

\subsection{Mass distributions}

The $\pi \pi, K K$, and $l l$ mass spectra for two representative $t$ ranges, together with the results of the fits discussed below, are shown in Fig. 6.

The acceptance-corrected $\pi \pi$ mass spectra for the $\rho^{0}$-candidate sample were fitted in the range $0.55<M_{\pi \pi}<1.3 \mathrm{GeV}$ using the Söding parameterisation [38]:

$$
\frac{\mathrm{d} \sigma}{\mathrm{d} M_{\pi \pi}}=A_{\rho^{0}}^{2}\left|\frac{\sqrt{M_{\pi \pi} M_{\rho^{0}} \Gamma_{\rho^{0}}}}{M_{\pi \pi}^{2}-M_{\rho^{0}}^{2}+i M_{\rho^{0}} \Gamma_{\rho^{0}}}+B / A_{\rho^{0}}\right|^{2}+f_{P S}\left(M_{\pi \pi}\right),
$$

where $B$ is the non-resonant amplitude (taken to be constant and real), $A_{\rho^{0}}$ is the normalisation of the resonant amplitude, $M_{\rho^{0}}$ is the $\rho^{0}$ mass and $\Gamma_{\rho^{0}}$ is the momentum-dependent $\rho^{0}$ width [39]. The additional term $f_{P S}=A_{P S}\left(1+1.5 M_{\pi \pi}\right)$ [10] was used to account for residual background from the non-diffractive $\gamma p \rightarrow$ anything and the diffractive $\gamma p \rightarrow X Y$ processes. The $\chi^{2} / n d f$ for all the fits is satisfactory. The ratio $\left|B / A_{\rho^{0}}\right|$ decreases from 
$0.13 \pm 0.02 \mathrm{GeV}^{-1 / 2}$ at $-t=1.3 \mathrm{GeV}^{2}$ to $0.05 \pm 0.03 \mathrm{GeV}^{-1 / 2}$ at $-t=2.3 \mathrm{GeV}^{2}$ and is consistent with zero above $-t=2.5 \mathrm{GeV}^{2}$. The background contribution in the range $0.6<M_{\pi \pi}<1.3 \mathrm{GeV}$, given by the integral of the function $f_{P S}$, increases from about $8 \pm 2 \%$ at $-t=1.3 \mathrm{GeV}^{2}$ to $17 \pm 4 \%$ at $-t=2.7 \mathrm{GeV}^{2}$ and is consistent with $15 \%$ above $-t=3.2 \mathrm{GeV}^{2}$.

The detector efficiency does not vary over the region of the $\phi$ and $J / \psi$ peaks. The $\phi$ and $J / \psi$ signals were, therefore, extracted from the uncorrected mass spectra. The $K K$ mass spectra for the sample of $\phi$ candidates were fitted in the range $0.99<M_{K K}<1.15 \mathrm{GeV}$ with the sum of a Breit-Wigner function convoluted with a Gaussian resolution function for the signal, and the function $\left(M_{K K}-2 M_{K}\right)^{\delta}$ for the background. The $\chi^{2} / n d f$ for all the fits is satisfactory. The background contribution in the range $0.99<M_{K K}<1.06 \mathrm{GeV}$ decreases from $25 \pm 2 \%$ at $-t=1.3 \mathrm{GeV}^{2}$ to $3 \pm 2 \%$ above $-t=4 \mathrm{GeV}^{2}$.

The $l l$ mass spectra for the $J / \psi$ candidate sample in the dilepton-invariant-mass range $2.6<M_{l l}<3.4 \mathrm{GeV}$ were fitted with the sum of a Gaussian (for the muon decay channel), a bremsstrahlung function convoluted with a Gaussian (for the electron decay channel), and a background term linear in $M_{l l}$. No muon or electron identification was performed; the relative contributions of muon and electron pairs were taken from the MC simulation. The electron mass w was used in calculating $M_{l l}$. The $\chi^{2} / n d f$ for all the fits is satisfactory. The background contribution in the range $2.7<M_{l l}<3.2 \mathrm{GeV}$ is $25 \pm 2 \%$, approximately independent of the $t$ range under study.

Since the backgrounds depend on $t, W, \cos \theta_{h}$ and $\varphi_{h}$, each of the results presented in the following sections was obtained by repeating the fits to the invariant mass distributions in each kinematic bin.

\subsection{Measurement of $\mathrm{d} \sigma_{\gamma p \rightarrow V Y} / \mathrm{d} t$}

Tables 1 - 3 and Fig. 7 show the differential cross sections for the $\rho^{0}, \phi$ and $J / \psi$ mesons obtained from the present data together with results obtained in the ZEUS 1995 [10] study of the lower $-t$ region. In that analysis, the cross section was determined for $M_{Y}^{2} / W^{2}<0.01$. In the kinematic region $-t \approx 1 \mathrm{GeV}^{2}$ and $W \approx 100 \mathrm{GeV}$, this limit is numerically equivalent to that used in the present study, so that the results may be directly compared. The two measurements are consistent in the region of overlap.

The cross sections are well described by a power-law dependence, as expected for a hard production mechanism [9]. A fit to the present data with the function $A \cdot(-t)^{-n}$ gives $n=3.21 \pm 0.04$ (stat.) \pm 0.15 (syst.) for the $\rho^{0}, n=2.7 \pm 0.1$ (stat.) \pm 0.2 (syst.) for the $\phi$, and $n=1.7 \pm 0.2$ (stat.) \pm 0.3 (syst.) for the $J / \psi$ meson.

\footnotetext{
$\overline{4}$ The results do not change when the muon mass is used.
} 
The measured values of $n$ for the $\rho^{0}$ and $\phi$ mesons are significantly smaller than the lower limit $n \approx 3.8$ predicted by Ivanov et al. [17]. The value of $n$ for the $J / \psi$ meson agrees with the Bartels et al. [16] BFKL prediction of 1.8. The BFKL prediction of Forshaw and Poludniowski [18], for the most natural choice of $\alpha_{s}=0.2$ and $W_{0}^{2}=M_{V}^{2}$, gives $\chi^{2} / n d f=0.5$ for all three mesons and is shown in Fig. 8. These measurements establish that perturbative QCD calculations are able to describe the large $-t$ regime. The dip at $-t \approx M_{V}^{2}$ predicted by the two-gluon exchange model of Forshaw and Ryskin [13] is not seen [18] in the data for light mesons, indicating that this model is not applicable in the $-t \approx M_{V}^{2}$ region.

Note that $n$ depends slightly on the $x$ range used in the cross-section definition both in the experiment and in the theory. Hence the values quoted in this paper apply only to the cross section integrated over $x>0.01$.

\subsection{Ratios of cross sections}

The cross-section ratios

$$
r_{\phi} \equiv \frac{\mathrm{d} \sigma_{\gamma p \rightarrow \phi Y}}{\mathrm{~d} t} / \frac{\mathrm{d} \sigma_{\gamma p \rightarrow \rho^{0} Y}}{\mathrm{~d} t}
$$

and

$$
r_{J / \psi} \equiv \frac{\mathrm{d} \sigma_{\gamma p \rightarrow J / \psi Y}}{\mathrm{~d} t} / \frac{\mathrm{d} \sigma_{\gamma p \rightarrow \rho^{0} Y}}{\mathrm{~d} t}
$$

are shown in Tables 4 and 5 and in Fig. 9, together with the values given in Eqs. (3), (4) and (5). A clear increase of $r_{\phi}$ and $r_{J / \psi}$ with increasing $-t$ is observed.

The dependence of $r_{\phi}$ and $r_{J / \psi}$ on $t$ suggests that the production mechanism may become flavour independent at larger $-t$ values than covered in this analysis. In the framework of $\mathrm{pQCD}$, this observation supports the hypothesis that the quark mass appearing in the $\gamma V$ coupling should be the constituent quark mass, since, in this case, the asymptotic value of $r_{\phi}$ should be reached at $-t \gg M_{\rho^{0}}^{2}, M_{\phi}^{2}$. If the quark mass is interpreted as the current quark mass, $r_{\phi}$ is expected to approach its asymptotic value at much smaller $-t$ values.

The cross-section ratios given in Eq. (5), expected for the production of mesons in the helicity-0 state by transverse photons at large $t[15,17]$, are the same as those expected from pQCD for the elastic production of mesons in the helicity-0 state by longitudinal photons at large $Q^{2}[8,40]$. The cross-section ratios for production of mesons in the helicity- \pm 1 states are expected to be larger than those for production of mesons in the helicity-0 state. Figure 10 compares the $t$ and $Q^{2}$ dependences of the ratios for the 
proton-dissociative and for the elastic process, respectively; $r_{\phi}$ lies systematically above $\sigma_{\gamma^{*} p \rightarrow \phi p} / \sigma_{\gamma^{*} p \rightarrow \rho^{0} p}[41,42]$ but is compatible within the experimental uncertainties, while $r_{J / \psi}$ for $-t>3 \mathrm{GeV}^{2}$ is about a factor of five larger than $\sigma_{\gamma^{*} p \rightarrow J / \psi p} / \sigma_{\gamma^{*} p \rightarrow \rho^{0} p}$ for $Q^{2}>$ $3 \mathrm{GeV}^{2}$ as measured by ZEUS [43] and H1 [44,45] Ð. The helicity analysis, described in Section 9.6, shows that the production of mesons in the helicity- \pm 1 states dominates in the large $-t$ region.

\subsection{The slope of the Pomeron trajectory}

The cross section given by Eq. (1), integrated over $M_{Y}$, can be expressed as

$$
\frac{\mathrm{d} \sigma_{\gamma p \rightarrow V Y}}{\mathrm{~d} t}=F(t) W^{4 \alpha(t)-4}
$$

where $F(t)$ is a function of $t$ only. Assuming a linear form for the Pomeron trajectory implies

$$
\frac{\mathrm{d} \sigma_{\gamma p \rightarrow V Y}}{\mathrm{~d} t}=F(t) W^{4 \alpha(0)+4 \alpha^{\prime} t-4}=F(t) G(W) W^{4 \alpha^{\prime} t},
$$

where $G(W)$ is a function of $W$ only.

The absolute $W$ dependence, and hence $\alpha(t)$, cannot be measured using the present data because of the large anti-correlated systematic uncertainties of the PT acceptance as a function of $W$. However, these uncertainties do not affect the measurement of the relative changes of the $W$ dependence as a function of $t$, which are sensitive to $\alpha^{\prime}$. The slope of the Pomeron trajectory for the $\rho^{0}$ meson was obtained by fitting the form given by Eq. (13) to the measured cross sections using three $W$ times six $t$ bins. A two-dimensional fit gave $\alpha^{\prime}=$ $-0.02 \pm 0.05$ (stat.) ${ }_{-0.08}^{+0.04}$ (syst.) $\mathrm{GeV}^{-2}$ with $\chi^{2} / n d f=4.6 / 9$. A similar fit for the $\phi$ meson, performed in three $W$ times five $t$ bins, gave $\alpha^{\prime}=-0.06 \pm 0.12$ (stat.) ${ }_{-0.09}^{+0.05}$ (syst.) $\mathrm{GeV}^{-2}$ with $\chi^{2} / n d f=3.4 / 7$. There were insufficient data to perform a similar analysis on the $J / \psi$ sample.

The consistency of the $\alpha^{\prime}$ values with zero implies that the $W$ dependence does not vary with $t$, and vice versa. This conclusion is manifest when looking at the $W$ dependence of the ratio of differential cross sections

$$
\frac{\frac{\mathrm{d} \sigma(W)}{\mathrm{d} t}}{\left.\frac{\mathrm{d} \sigma(W)}{\mathrm{d} t}\right|_{t=t_{0}}} \propto W^{4 \alpha^{\prime}\left(t-t_{0}\right)},
$$

shown in Figs. 11 and 12. Values of $-t_{0}=1.3 \mathrm{GeV}^{2}$ were chosen for the $\rho^{0}$ and $-t_{0}=$ $1.5 \mathrm{GeV}^{2}$ for the $\phi$ case.

\footnotetext{
${ }^{5}$ The $J / \psi$ data from $\mathrm{H} 1$ have been scaled from $W=90 \mathrm{GeV}$ to $W=75 \mathrm{GeV}$ and the $\rho^{0}$ data calculated at the same $Q^{2}$ values using a fit of the form $\left(Q^{2}+M_{\rho^{0}}^{2}\right)^{-n}[46]$
} 
Figure 13 compares the values of $\alpha^{\prime}$ measured in this analysis with those obtained at lower $-t$. For both $\rho^{0}$ and $\phi$ mesons, the values of $\alpha^{\prime}$ for $-t>1.3 \mathrm{GeV}^{2}$ are smaller than the value $\alpha^{\prime}=0.25 \mathrm{GeV}^{-2}$ characteristic of soft hadronic processes at $-t<0.5 \mathrm{GeV}^{2}$ and also below the values $\alpha^{\prime}=0.125 \pm 0.038 \mathrm{GeV}^{-2}, \alpha^{\prime}=0.158 \pm 0.028 \mathrm{GeV}^{-2}$ and $\alpha^{\prime}=0.113 \pm 0.018 \mathrm{GeV}^{-2}$ obtained for $-t<1.5 \mathrm{GeV}^{2}$, from fits $[10,11]$ to the elastic photoproduction of $\rho^{0}, \phi$ and $J / \psi$ mesons, respectively. This observation indicates that $\alpha^{\prime}$ decreases with increasing $-t$. The small values of $\alpha^{\prime}$ obtained in this analysis are consistent with the flatness of the Pomeron trajectory observed by UA8 [47] at large $-t$ and with the expectation of the anomalous Regge exchange model by Kochelev et al. [12]. They are also consistent with the expectation of $\alpha^{\prime}=0$ characteristic of the two-gluonexchange models $[13,15,17,18]$ and the expectation of $\alpha^{\prime} \lesssim 0.1 \mathrm{GeV}^{-2}$ of the BFKL model of Bartels et al. [16].

\subsection{Decay angular distributions}

The $r_{00}^{04}$ and $r_{1-1}^{04}$ spin density matrix elements for the $\rho^{0}, \phi$, and $J / \psi$ were obtained by fitting Eqs. (8) and (9) to the background-subtracted and acceptance-corrected data in several $t$ ranges. The $t$ ranges and the results of the fits are given in Tables 6, 7 and 8. As an example, Fig. 14 shows the $\cos \theta_{h}$ and $\varphi_{h}$ distributions with the results of the fits in the highest $-t$ bin used in the helicity analysis for each of the three mesons. The flat $\varphi_{h}$ distribution shown by the dashed line disagrees $\left(\chi^{2} / n d f=42 / 7\right)$ with the $\rho^{0}$ data, indicating a violation of SCHC. The parameter $\operatorname{Re}\left[r_{10}^{04}\right]$ for the $\rho^{0}$ and $\phi$ mesons was determined using a two-dimensional fit of Eq. (7) in three and two $t$ intervals, respectively. The results of the fits are summarised in Tables 6 and 0 . The above results were also confirmed by a decay-angle analysis [48] performed in the transversity frame 0 . There were insufficient data to perform a two-dimensional fit for the $J / \psi$ meson.

The fitted values of $r_{00}^{04}, \operatorname{Re}\left[r_{10}^{04}\right]$, and $r_{1-1}^{04}$ for the $\rho^{0}$ meson are displayed in Fig. 15. The results are consistent with the ZEUS 1995 [10] measurements in the overlap region. The small values of $r_{00}^{04}$ indicate that the probability to produce $\rho^{0}$ mesons in the helicity-0 state from a photon in the helicity- \pm 1 states is $4 \pm 5 \%$ at $-t=3.35 \mathrm{GeV}^{2}$ and $9 \pm 6 \%$ at $-t=5.67 \mathrm{GeV}^{2}$. However, the non-zero value of $\operatorname{Re}\left[r_{10}^{04}\right]$ indicates a helicity single-flip contribution at the level of a few percent. The finite negative values of $r_{1-1}^{04}$ show clear evidence for a helicity-double-flip contribution.

The measurements for the $\phi$ meson, shown in Fig. 16, display similar features to those for the $\rho^{0}$ meson, although with a smaller statistical significance. The values of $r_{00}^{04}$ and

${ }^{6}$ The spin-quantisation axis in the transversity frame is chosen along the normal to the meson production frame. 
$r_{1-1}^{04}$ for the $J / \psi$ meson, shown in Fig. 17, are consistent with the SCHC hypothesis.

Although the BFKL calculation of Forshaw and Poludniowski [18] successfully describes the cross sections for all three mesons, it is not in accord with the observation of SCHC violation. This calculation forces SCHC by using a delta-function for the meson wavefunction.

If a more appropriate meson wave-function for the light mesons is used, the helicity of the final-state meson will depend on the modelling of the photon fluctuation into a $q \bar{q}$ pair. Within perturbation theory, a photon can only split into a $q \bar{q}$ pair in a chiral-even spin configuration. If, in addition, the quark appearing in the $\gamma V$ coupling is interpreted as a current quark, as assumed by Ginzburg and Ivanov [15], real photons will produce light mesons only in the helicity-0 state because of the chiral nature of the perturbative coupling in the massless limit. Not only does this model not agree with the cross section [10], but it is also ruled out by the helicity data. Ivanov et al. [17] have proposed that a large contribution of light mesons produced in the helicity- \pm 1 states could arise from $q \bar{q}$ fluctuations in a chiral-odd spin configuration. This model fails to describe the cross section and does not quantitatively describe the helicity structure.

\section{Summary}

Diffractive photoproduction of $\rho^{0}, \phi$ and $J / \psi$ mesons with proton dissociation has been measured in the ZEUS detector at HERA using data at $W \approx 100 \mathrm{GeV}$ for $-t$ values up to $12 \mathrm{GeV}^{2}$. Expressing the differential cross section as a power-law, $\mathrm{d} \sigma_{\gamma p \rightarrow V Y} / \mathrm{d} t \propto(-t)^{-n}$, yields a value for $n$ that decreases with increasing mass of the meson. The resulting exponents are: $3.21 \pm 0.04$ (stat.) \pm 0.15 (syst.) for $\rho^{0}$ production; $2.7 \pm 0.1$ (stat.) \pm 0.2 (syst.) for $\phi$ production; and $1.7 \pm 0.2$ (stat.) \pm 0.3 (syst.) for $J / \psi$ production. The BFKL calculation of Bartels et al. [16] agrees with the $J / \psi$ data. An extension of this model by Forshaw and Poludniowski [18] is able to describe the data for all three mesons.

The cross-section ratios for the $\phi$ and $J / \psi$ with respect to the $\rho$ as a function of $t$ increase with $-t$. In the context of $\mathrm{pQCD}$, this observation suggests that the production mechanism may become flavour independent at larger $-t$ values than those covered in this analysis.

The decay-angle analysis for the $\rho^{0}$ and $\phi$ mesons indicates that the SCHC hypothesis does not hold, since both single and double helicity-flip contributions are observed. No available pQCD calculation is able to describe this result quantitatively.

The effective slope of the Pomeron trajectory, $\alpha^{\prime}$, was determined by studying the relative changes of the $W$ dependence of the cross-section as a function of $t$ for the $\rho^{0}$ and $\phi$ 
mesons. The slopes are $\alpha^{\prime}=-0.02 \pm 0.05$ (stat.) ${ }_{-0.08}^{+0.04}$ (syst.) $\mathrm{GeV}^{-2}$ for the $\rho^{0}$ meson and $\alpha^{\prime}=-0.06 \pm 0.12$ (stat.) ${ }_{-0.09}^{+0.05}$ (syst.) $\mathrm{GeV}^{-2}$ for the $\phi$ meson. These values are lower than the value characteristic of low $-t$ hadronic scattering $\left(\alpha^{\prime}=0.25 \mathrm{GeV}^{-2}\right)$ and lower than those obtained in elastic vector-meson photoproduction at HERA at lower $-t[10,11]$. They are in agreement with pQCD expectations $[16,17]$ and are consistent with the flatness of the Pomeron trajectory at large $-t$ observed by UA8 [47]. These results imply that large values of $-t$ can provide a suitable hard scale for perturbative QCD calculations.

\section{Acknowledgments}

We thank the DESY directorate for their strong support and encouragement. The remarkable achievements of the HERA machine group were essential for the successful completion of this work and are gratefully acknowledged. We are grateful for the support of the DESY computing and network services. The design, construction and installation of the ZEUS detector has been made possible by the efforts and ingenuity of many people from DESY and the home institutes who are not listed as authors. We are grateful to J.R. Forshaw and G. Poludniowski for providing the results of their calculation and to J. Bartels and D.Yu Ivanov for useful discussion. 


\section{References}

[1] J.A. Crittenden, Exclusive Production of Neutral Vector Mesons at the

Electron-Proton Collider HERA, Springer Tracts in Modern Physics, Vol. 140.

Springer, Berlin, Germany, 1997;

H. Abramowicz and A. Caldwell, Rev. Mod. Phys. 71, 1275 (1999).

[2] A. Donnachie and P.V. Landshoff, Nucl. Phys. B 231, 189 (1984).

[3] J.R. Cudell, K. Kang and S.K. Kim, Phys. Lett. B 395, 311 (1997).

[4] P.D.B. Collins, An Introduction to Regge Theory and High Energy Physics. Cambridge University Press, 1977.

[5] A. Donnachie and P.V. Landshoff, Phys. Lett. B 470, 243 (1999);

A. Donnachie and P.V. Landshoff, Phys. Lett. B 478, 146 (2000);

E. Martynov, E. Predazzi and A. Prokudin, Preprint hep-ph/0112242, 2001. Subm. to Eur. Phys. J.

[6] J.J. Sakurai, Ann. Phys. 11, 1 (1960);

J.J. Sakurai, Phys. Rev. Lett. 22, 981 (1969).

[7] M.G. Ryskin, Z. Phys. C 57, 89 (1993).

[8] S.J. Brodsky et al., Phys. Rev. D 50, 3134 (1994).

[9] L. Frankfurt and M. Strikman, Phys. Rev. Lett. 63, 1914 (1989);

A.H. Mueller and W.K. Tang, Phys. Lett. B 284, 123 (1992).

[10] ZEUS Coll., J. Breitweg et al., Eur. Phys. J. C 14, 213 (2000).

[11] ZEUS Coll., S. Chekanov et al., Preprint DESY-02-008 (hep-ex-0201043), DESY, 2002. Subm. to Eur. Phys. J.

[12] N.I. Kochelev et al., Nucl. Phys. Proc. Suppl. 99 A, 24 (2001).

[13] J.R. Forshaw and M.G. Ryskin, Z. Phys. C 68, 137 (1995).

[14] D.Yu. Ivanov, Phys. Rev. D 53, 3564 (1996).

[15] I.F. Ginzburg and D.Yu. Ivanov, Phys. Rev. D 54, 5523 (1996).

[16] J. Bartels et al., Phys. Lett. B 375, 301 (1996).

[17] D.Yu. Ivanov et al., Phys. Lett. B 478, 101 (2000). Erratum in Phys. Lett. B 498, 295 (2001).

[18] J.R Forshaw and G. Poludniowski, Preprint hep-ph/0107068, 2001.

[19] M. Glück, E. Reya and A. Vogt, Eur. Phys. J. C 5, 461 (1998). 
[20] E.A. Kuraev, L.N. Lipatov and V.S. Fadin, Sov. Phys. JETP 45, 199 (1977);

Ya.Ya. Balitskĭ and L.N. Lipatov, Sov. J. Nucl. Phys. 28, 822 (1978).

[21] F.J. Gilman et al., Phys. Lett. B 31, 387 (1970).

[22] Particle Data Group, D.E. Groom et al., Eur. Phys. J. C 15, 1 (2000).

[23] ZEUS Coll., U. Holm (ed.), The ZEUS Detector. Status Report (unpublished), DESY (1993), available on http://www-zeus.desy.de/bluebook/bluebook.html.

[24] N. Harnew et al., Nucl. Inst. Meth. A 279, 290 (1989);

B. Foster et al., Nucl. Phys. Proc. Suppl. B 32, 181 (1993);

B. Foster et al., Nucl. Inst. Meth. A 338, 254 (1994).

[25] M. Derrick et al., Nucl. Inst. Meth. A 309, 77 (1991);

A. Andresen et al., Nucl. Inst. Meth. A 309, 101 (1991);

A. Caldwell et al., Nucl. Inst. Meth. A 321, 356 (1992);

A. Bernstein et al., Nucl. Inst. Meth. A 336, 23 (1993).

[26] ZEUS Coll., J. Breitweg et al., Z. Phys. C 75, 421 (1997).

[27] J. Andruszków et al., Acta Phys. Pol. B 32, 2025 (2001).

[28] K. Schilling and G. Wolf, Nucl. Phys. B 61, 381 (1973).

[29] M. Kasprzak, Inclusive Properties of Diffractive and Non-diffractive

Photoproduction at HERA. Ph.D. Thesis, Warsaw University, Warsaw, Poland, Report DESY F35D-96-16, DESY, 1996.

[30] L. Adamczyk, Vector Meson Photoproduction at Large Momentum Transfer at HERA. Ph.D. Thesis, University of Mining and Metallurgy, Cracow, Poland, Report DESY-THESIS-1999-045, DESY, 1999.

[31] B. List and A. Mastroberardino, Proc. Workshop on Monte Carlo Generators for HERA Physics, p. 396. DESY, Hamburg, Germany (1999). Also in preprint DESY-PROC-1999-02, available on http://www.desy.de/ ${ }^{\text {heramc/. }}$

[32] R.L. Cool et al., Phys. Rev. Lett. 48, 1451 (1982);

R. Hagedorn, Riv. Nuovo Cim. 6, 1 (1984).

[33] T. Sjöstrand, Comp. Phys. Comm. 82, 74 (1994).

[34] R. Brun et al., GEAnt3, Technical Report CERN-DD/EE/84-1, CERN, 1987.

[35] K.H. Klimek, Cross Section Measurement of Vector Meson Quasi-Photoproduction at High Four-Momentum Transfer Using the ZEUS Detector at the HERA Collider. Ph.D. Thesis, Institute of Nuclear Physics, Cracow, Poland, Report DESY-THESIS-2001-053, DESY, 2001.

[36] V.M. Budnev et al., Phys. Rep. 15C, 181 (1974). 
[37] D.P. Roy and R.G. Roberts, Nucl. Phys. B 77, 240 (1974).

[38] P. Söding, Phys. Lett. 19, 702 (1966).

[39] J.D. Jackson, Nuovo Cimento 34, 1644 (1964).

[40] H. Abramowicz, L. Frankfurt and M. Strikman, Surv. High Energy Phys. 11, 51 (1997).

[41] H1 Coll., C. Adloff et al., Phys. Lett. B 483, 360 (2000).

[42] ZEUS Coll., J. Breitweg et al., Phys. Lett. B 487, 273 (2000).

[43] ZEUS Coll., J. Breitweg et al., Eur. Phys. J. C 6, 603 (1999).

[44] H1 Coll., C. Adloff et al., Eur. Phys. J. C 13, 371 (2000).

[45] H1 Coll., C. Adloff et al., Eur. Phys. J. C 10, 373 (1999).

[46] A.B. Meyer, private communication.

[47] UA8 Coll., A. Brandt et al., Nucl. Phys. B 514, 3 (1998).

[48] A. Kowal, Helicity Analysis of Vector Mesons Produced in Proton-dissociative Diffractive Photoproduction at Large Momentum Transfer at HERA. Ph.D. Thesis, University of Mining and Metallurgy, Cracow, Poland, 2002. Unpublished.

[49] ZEUS Coll., M. Derrick et al., Phys. Lett. B 377, 259 (1996).

[50] ZEUS Coll., M. Derrick et al., Phys. Lett. B 380, 220 (1996).

[51] ZEUS Coll., J. Breitweg et al., Z. Phys. C 75, 215 (1997). 


\begin{tabular}{|c|c|c|}
\hline \multicolumn{3}{|c|}{ ZEUS 1996-97 $\gamma p \rightarrow \rho^{0} Y$} \\
\hline $\begin{array}{c}-t \text { range } \\
\left(\mathrm{GeV}^{2}\right)\end{array}$ & $\begin{array}{c}\langle-t\rangle \\
\left(\mathrm{GeV}^{2}\right)\end{array}$ & $\begin{array}{c}\mathrm{d} \sigma / \mathrm{d}|t| \\
\left(n b / \mathrm{GeV}^{2}\right)\end{array}$ \\
\hline $1.20-1.50$ & 1.33 & $163 \pm 6_{-19}^{+14}{ }_{-12}^{+7}$ \\
\hline $1.50-2.00$ & 1.71 & $80.1 \pm 2.5_{-8.5}^{+5.5}{ }_{-8.5}^{+2.0}$ \\
\hline $2.00-2.50$ & 2.22 & $36.9 \pm 1.4_{-2.8}^{+3.4}{ }_{-2.6}^{+0.4}$ \\
\hline $2.50-3.00$ & 2.72 & $17.5 \pm 0.9_{-1.5}^{+1.3}{ }_{-1.7}^{+0.1}$ \\
\hline $3.00-3.50$ & 3.23 & $10.7 \pm 0.7_{-0.7}^{+0.8}{ }_{-1.2}^{+0.3}$ \\
\hline $3.50-4.00$ & 3.73 & $6.98 \pm 0.59_{-0.41}^{+0.81}{ }_{-0.97}^{+0.39}$ \\
\hline $4.00-5.00$ & 4.43 & $3.39 \pm 0.27_{-0.50}^{+0.78}{ }_{-0.57}^{+0.23}$ \\
\hline $5.00-6.85$ & 5.75 & $1.41 \pm 0.15_{-0.49}^{+0.35}{ }_{-0.25}^{+0.20}$ \\
\hline $6.85-10.0$ & 8.04 & $0.46 \pm 0.07_{-0.22}^{+0.17}{ }_{-0.13}^{+0.08}$ \\
\hline
\end{tabular}

Table 1: The differential cross section, $\mathrm{d} \sigma_{\gamma p \rightarrow \rho^{0} Y} / \mathrm{d}|t|$, for $x>0.01$ and $80<W<$ $120 \mathrm{GeV}$. The first uncertainty is the statistical, the second the systematic and the last is due to the modelling of the proton-dissociation process. The normalisation uncertainty of $10 \%$ is not included.

\begin{tabular}{|c|c|c|}
\hline \multicolumn{3}{|c|}{ ZEUS 1996-97 $\gamma p \rightarrow \phi Y$} \\
\hline $\begin{array}{c}-t \text { range } \\
\left(\mathrm{GeV}^{2}\right)\end{array}$ & $\begin{array}{c}\langle-t\rangle \\
\left(\mathrm{GeV}^{2}\right)\end{array}$ & $\begin{array}{c}\mathrm{d} \sigma / \mathrm{d}|t|) \\
\left(\mathrm{nb} / \mathrm{GeV}^{2}\right)\end{array}$ \\
\hline $1.20-1.55$ & 1.36 & $19.2 \pm 1.1_{-2.1}^{+2.5}{ }_{-1.7}^{+1.0}$ \\
\hline $1.55-1.90$ & 1.71 & $12.4 \pm 0.7_{-1.1}^{+1.8}{ }_{-0.9}^{+0.3}$ \\
\hline $1.90-2.30$ & 2.08 & $7.43 \pm 0.53_{-0.72}^{+0.68}{ }_{-0.41}^{+0.30}$ \\
\hline $2.30-2.70$ & 2.48 & $4.63 \pm 0.40_{-0.43}^{+0.49}{ }_{-0.08}^{+0.17}$ \\
\hline $2.70-3.20$ & 2.93 & $2.55 \pm 0.26_{-0.29}^{+0.30}{ }_{-0.08}^{+0.15}$ \\
\hline $3.20-3.80$ & 3.47 & $1.55 \pm 0.20_{-0.25}^{+0.33}{ }_{-0.07}^{+0.13}$ \\
\hline $3.80-4.80$ & 4.24 & $0.89 \pm 0.18_{-0.14}^{+0.31}{ }_{-0.07}^{+0.13}$ \\
\hline $4.80-6.50$ & 5.53 & $0.36 \pm 0.08_{-0.12}^{+0.09}{ }_{-0.06}^{+0.10}$ \\
\hline
\end{tabular}

Table 2: The differential cross section, $\mathrm{d} \sigma_{\gamma p \rightarrow \phi Y} / \mathrm{d}|t|$, for $x>0.01$ and $80<W<$ $120 \mathrm{GeV}$. The first uncertainty is the statistical, the second the systematic and the last is due to the modelling of the proton-dissociation process. The normalisation uncertainty of $10 \%$ is not included. 


\begin{tabular}{|c|c|c|}
\hline \multicolumn{3}{|c|}{ ZEUS 1996-97 $\gamma p \rightarrow J / \psi Y$} \\
\hline $\begin{array}{c}-t \text { range } \\
\left(\mathrm{GeV}^{2}\right)\end{array}$ & $\begin{array}{c}\langle-t\rangle \\
\left(\mathrm{GeV}^{2}\right)\end{array}$ & $\begin{array}{c}\mathrm{d} \sigma / \mathrm{d}|t| \\
\left(n b / \mathrm{GeV}^{2}\right)\end{array}$ \\
\hline $1.20-1.50$ & 1.34 & $16.0 \pm 2.4_{-3.4}^{+1.6}{ }_{-4.8}^{+1.6}$ \\
\hline $1.50-2.00$ & 1.74 & $9.5 \pm 1.5_{-1.4}^{+1.4}{ }_{-1.2}^{+0.3}$ \\
\hline $2.00-2.50$ & 2.24 & $8.3 \pm 1.5_{-1.6}^{+1.6}{ }_{-2.4}^{+0.3}$ \\
\hline $2.50-3.25$ & 2.84 & $4.98 \pm 0.95_{-0.90}^{+0.89}{ }_{-0.63}^{+0.38}$ \\
\hline $3.25-4.50$ & 3.81 & $2.5 \pm 0.6_{-1.0_{-0.4}^{+0.8}}^{+0.4}$ \\
\hline $4.50-6.50$ & 5.37 & $1.33 \pm 0.45_{-0.41}^{+0.80}{ }_{-0.39}^{+0.16}$ \\
\hline
\end{tabular}

Table 3: The differential cross section, $\mathrm{d} \sigma_{\gamma p \rightarrow J / \psi Y} / \mathrm{d}|t|$, for $x>0.01$ and $80<W<120 \mathrm{GeV}$. The first uncertainty is the statistical, the second the systematic and the last is due to the modelling of the proton-dissociation process. The normalisation uncertainty of $10 \%$ is not included.

\begin{tabular}{|c|c|c|}
\hline \multicolumn{3}{|c|}{ ZEUS 1996-97 } \\
\hline$-t$ range & $\langle-t\rangle$ & $\frac{\mathrm{d} \sigma}{\mathrm{d} t}(\gamma p \rightarrow \phi Y) / \frac{\mathrm{d} \sigma}{\mathrm{d} t}\left(\gamma p \rightarrow \rho^{0} Y\right)$ \\
\hline $1.20-1.55$ & 1.35 & $0.124 \pm 0.008_{-0.012}^{+0.020}{ }_{-0.013}^{+0.014}$ \\
\hline $1.55-1.90$ & 1.71 & $0.156 \pm 0.011_{-0.010}^{+0.023}{ }_{-0.013}^{+0.014}$ \\
\hline $1.90-2.30$ & 2.08 & $0.176 \pm 0.015_{-0.017}^{+0.021}{ }_{-0.010}^{+0.011}$ \\
\hline $2.30-2.70$ & 2.48 & $0.167 \pm 0.016_{-0.013}^{+0.013}{ }_{-0.006}^{+0.006}$ \\
\hline $2.70-3.20$ & 2.93 & $0.185 \pm 0.021_{-0.034}^{+0.014}{ }_{-0.013}^{+0.013}$ \\
\hline $3.20-3.80$ & 3.47 & $0.189 \pm 0.027_{-0.038}^{+0.020}{ }_{-0.020}^{+0.021}$ \\
\hline $3.80-4.80$ & 4.23 & $0.223 \pm 0.047_{-0.042}^{+0.057}{ }_{-0.033}^{+0.034}$ \\
\hline $4.80-6.50$ & 5.49 & $0.225 \pm 0.052_{-0.060}^{+0.055}{ }_{-0.052}^{+0.055}$ \\
\hline
\end{tabular}

Table 4: The ratio of the differential cross sections, $\mathrm{d} \sigma / \mathrm{d} t$, for $\phi$ to $\rho^{0}$ protondissociative photoproduction for $x>0.01$ and $80<W<120 \mathrm{GeV}$. The first uncertainty is the statistical, the second the systematic and the last is due to the modelling of the proton-dissociation process. 


\begin{tabular}{|c|c|c|}
\hline \multicolumn{3}{|c|}{ ZEUS 1996-97 } \\
\hline$-t$ range & $\langle-t\rangle$ & $\frac{\mathrm{d} \sigma}{\mathrm{d} t}(\gamma p \rightarrow J / \psi Y) / \frac{\mathrm{d} \sigma}{\mathrm{d} t}\left(\gamma p \rightarrow \rho^{0} Y\right)$ \\
\hline $1.20-1.50$ & 1.33 & $0.099 \pm 0.015_{-0.019}^{+0.021}{ }_{-0.007}^{+0.010}$ \\
\hline $1.50-2.00$ & 1.71 & $0.119 \pm 0.019_{-0.017}^{+0.027}{ }_{-0.007}^{+0.007}$ \\
\hline $2.00-2.50$ & 2.22 & $0.226 \pm 0.042_{-0.037}^{+0.056}{ }_{-0.008}^{+0.005}$ \\
\hline $2.50-3.25$ & 2.82 & $0.325 \pm 0.063_{-0.059}^{+0.020}{ }_{-0.018}^{+0.013}$ \\
\hline $3.25-4.50$ & 3.75 & $0.409 \pm 0.097_{-0.121}^{+0.078}{ }_{-0.046}^{+0.038}$ \\
\hline $4.50-6.50$ & 5.28 & $0.72 \pm 0.25_{-0.22}^{+0.20}{ }_{-0.15}^{+0.11}$ \\
\hline
\end{tabular}

Table 5: The ratio of the differential cross sections, $\mathrm{d} \sigma / \mathrm{d} t$, for $J / \psi$ to $\rho^{0}$ protondissociative photoproduction for $x>0.01$ and $80<W<120 \mathrm{GeV}$. The first uncertainty is the statistical, the second the systematic and the last is due to the modelling of the proton-dissociation process.

\begin{tabular}{|c|c|c|c|c|}
\hline \multicolumn{5}{|c|}{ ZEUS 1996-97 $\gamma p \rightarrow \rho^{0} Y$} \\
\hline $\begin{array}{c}-t \text { range } \\
\left(\mathrm{GeV}^{2}\right)\end{array}$ & $\begin{array}{c}\langle-t\rangle \\
\left(\mathrm{GeV}^{2}\right)\end{array}$ & $r_{00}^{04}$ & $R e\left[r_{10}^{04}\right]$ & $r_{1-1}^{04}$ \\
\hline $1.1-1.7$ & 1.34 & $0.022 \pm 0.015_{-0.018}^{+0.016}$ & $0.054 \pm 0.012_{-0.010}^{+0.017}$ & $-0.143 \pm 0.014_{-0.031}^{+0.023}$ \\
\hline $1.7-2.9$ & 2.14 & $0.042 \pm 0.017_{-0.021}^{+0.018}$ & $0.045 \pm 0.013_{-0.010}^{+0.016}$ & $-0.158 \pm 0.017_{-0.022}^{+0.020}$ \\
\hline $2.9-4.0$ & 3.35 & $0.037 \pm 0.034_{-0.036}^{+0.032}$ & & $-0.129 \pm 0.032_{-0.050}^{+0.023}$ \\
\hline $4.0-12$ & 5.67 & $0.090 \pm 0.049_{-0.040}^{+0.035}$ & & $-0.252 \pm 0.047_{-0.023}^{+0.044}$ \\
\hline $2.9-12$ & 4.38 & & $0.047 \pm 0.020_{-0.017}^{+0.009}$ & \\
\hline
\end{tabular}

Table 6: The spin density matrix elements for $\gamma p \rightarrow \rho^{0} Y$ for $80<W<120 \mathrm{GeV}$. The first uncertainty is the statistical and the second the systematic.

\begin{tabular}{|c|c|c|c|c|}
\hline \multicolumn{5}{|c|}{ ZEUS 1996-97 $\gamma p \rightarrow \phi Y$} \\
\hline $\begin{array}{c}-t \text { range } \\
\left(\mathrm{GeV}^{2}\right)\end{array}$ & $\begin{array}{c}\langle-t\rangle \\
\left(\mathrm{GeV}^{2}\right)\end{array}$ & $r_{00}^{04}$ & $R e\left[r_{10}^{04}\right]$ & $r_{1-1}^{04}$ \\
\hline $1.2-1.7$ & 1.42 & $0.080 \pm 0.028_{-0.026}^{+0.020}$ & $0.053 \pm 0.021_{-0.024}^{+0.019}$ & $0.008 \pm 0.033_{-0.039}^{+0.011}$ \\
\hline $1.7-3.0$ & 2.20 & $0.066 \pm 0.025_{-0.021}^{+0.034}$ & & $-0.085 \pm 0.032_{-0.028}^{+0.023}$ \\
\hline $3.0-7.0$ & 4.03 & $-0.020 \pm 0.035_{-0.018}^{+0.034}$ & & $-0.108 \pm 0.058_{-0.030}^{+0.053}$ \\
\hline $1.7-7.0$ & 2.72 & & $0.057 \pm 0.018_{-0.007}^{+0.013}$ & \\
\hline
\end{tabular}

Table 7: The spin density matrix elements for $\gamma p \rightarrow \phi Y$ for $80<W<120 \mathrm{GeV}$. The first uncertainty is the statistical and the second the systematic. 


\begin{tabular}{|c|c|c|c|}
\hline \multicolumn{4}{|c|}{ ZEUS 1996-97 $\gamma p \rightarrow J / \psi Y$} \\
\hline $\begin{array}{c}-t \text { range } \\
\left(\mathrm{GeV}^{2}\right)\end{array}$ & $\begin{array}{c}\langle-t\rangle \\
\left(\mathrm{GeV}^{2}\right)\end{array}$ & $r_{00}^{04}$ & $r_{1-1}^{04}$ \\
\hline $1.1-1.8$ & 1.42 & $-0.28 \pm 0.26_{-0.08}^{+0.28}$ & $0.10 \pm 0.15_{-0.03}^{+0.16}$ \\
\hline $1.8-6.5$ & 3.01 & $0.22 \pm 0.30_{-0.11}^{+0.38}$ & $-0.11 \pm 0.16_{-0.04}^{+0.06}$ \\
\hline
\end{tabular}

Table 8: The spin density matrix elements for $\gamma p \rightarrow J / \psi Y$ for $80<W<$ $120 \mathrm{GeV}$. The first uncertainty is the statistical and the second the systematic. 

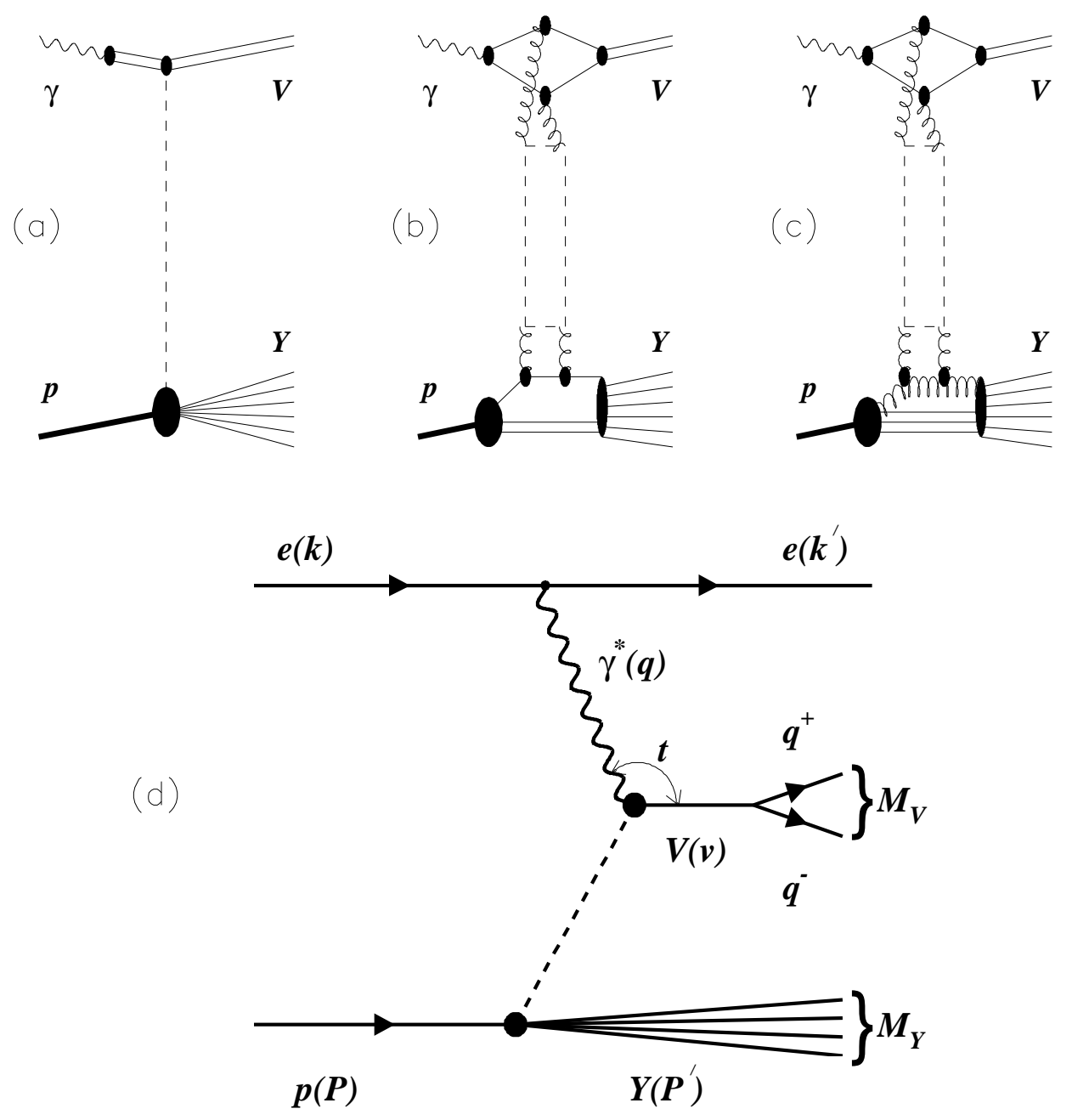

Figure 1: (a) $\gamma p \rightarrow V Y$ scattering in the VDM model. The photon fluctuates into a vector meson, which then scatters off the proton and the proton breaks up. (b,c) Example of $\gamma p \rightarrow V Y$ scattering in $p Q C D$ models. The photon fluctuates into a $q \bar{q}$ pair, which then scatters off a single quark (b) or gluon (c) in the proton by the exchange of two gluons or a gluon ladder. The scattered $q \bar{q}$ pair becomes a vector meson. The struck parton and the proton remnant fragment into a system of hadrons. (d) Schematic diagram of proton-dissociative vector-meson production in ep interactions, $\mathrm{ep} \rightarrow \mathrm{eVY}$. 


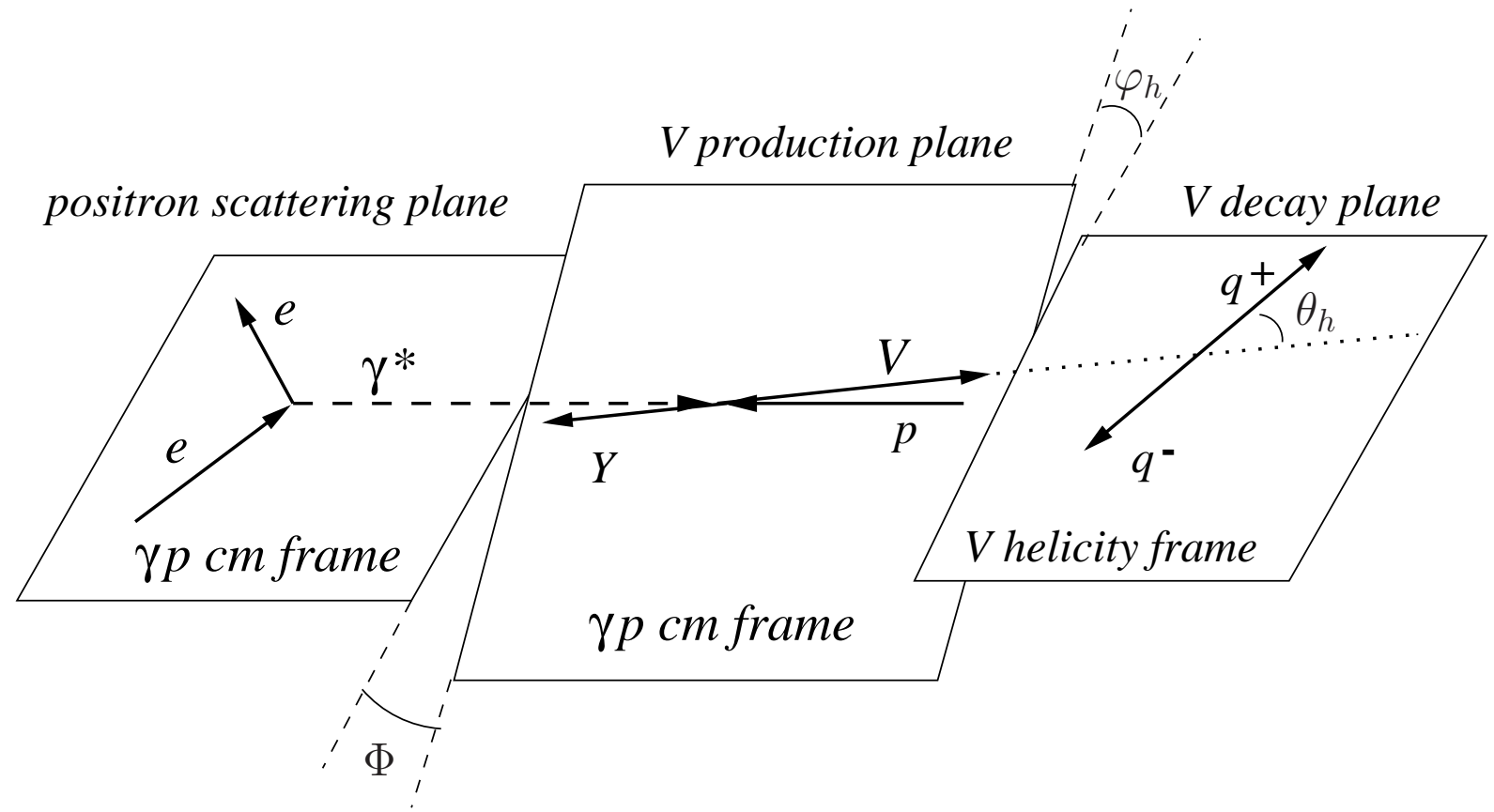

Figure 2: Illustration of the angles used to analyse the helicity states of a vector meson (for a decay into two particles, $V \rightarrow q^{+} q^{-}$). 
ZEUS
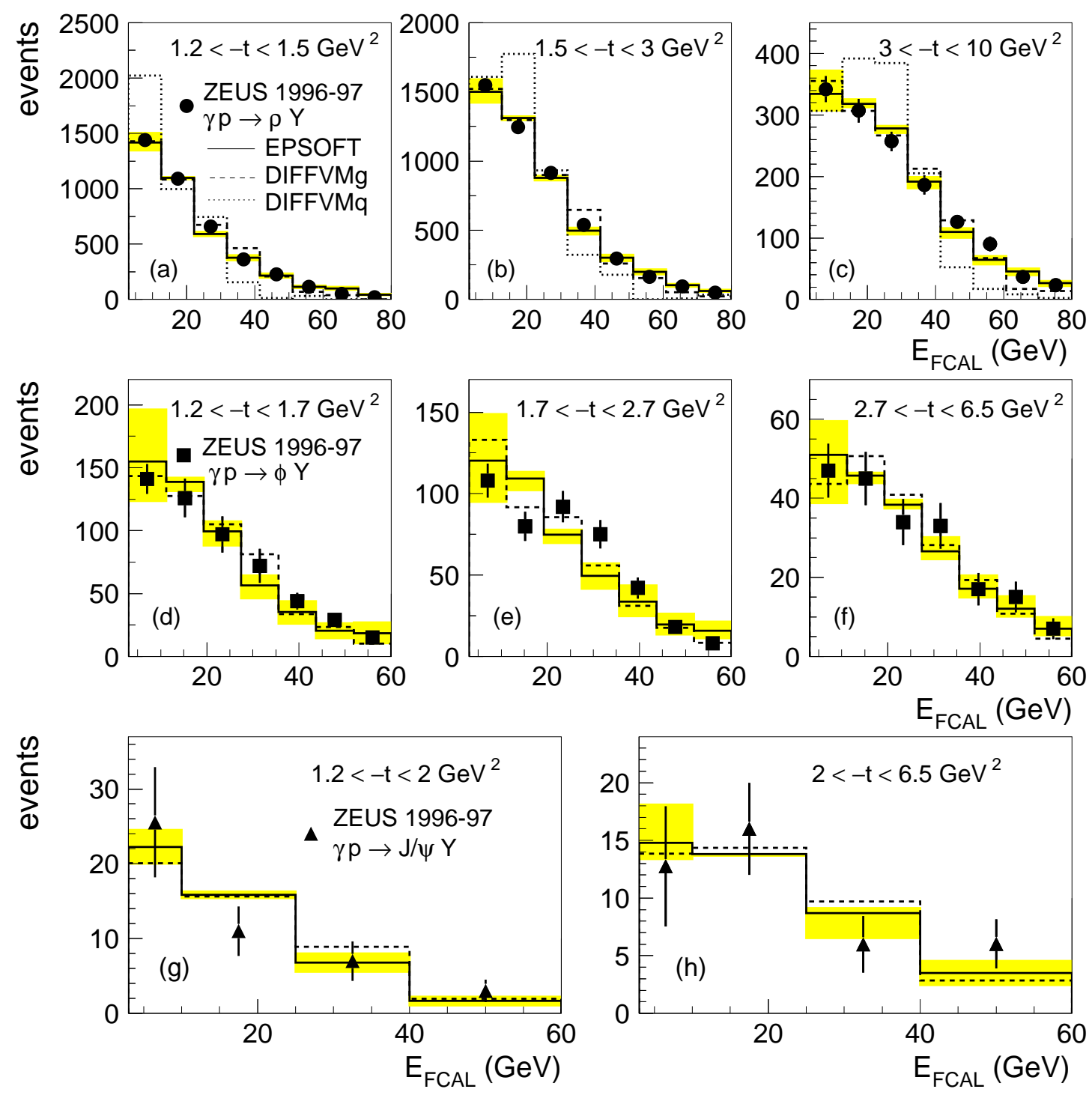

Figure 3: The distributions of the FCAL energy for: $(a, b, c) \rho^{0} ;(d, e, f) \phi ;(g, h)$ $J / \psi$ candidate samples in different $-t$ regions. The symbols represent the data and the histograms indicate the results of the simulation using EPSOFT (solid line), DIFFVMg (dashed lines) and DIFFVMq (dotted lines shown only for the $\rho^{0}$ ). The shaded bands represent the size of the correlated uncertainties due to the modelling of the proton dissociation in EPSOFT. 


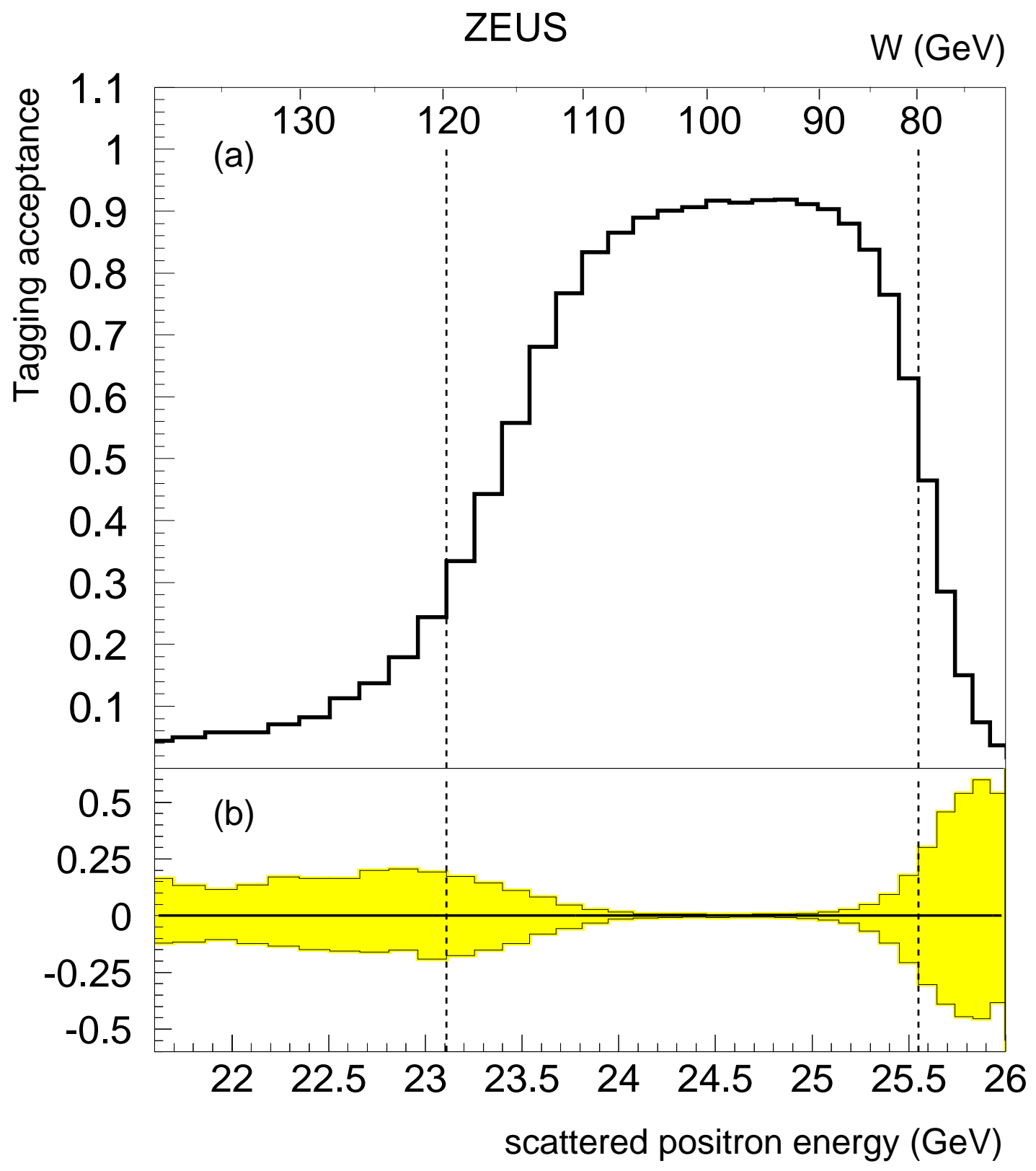

Figure 4: The photoproduction tagging acceptance as a function of the energy of the scattered positron calculated using simulated events generated according to the equivalent-photon approximation in the $Q^{2}<0.02 \mathrm{GeV}^{2}$ range. The shaded band represents the relative systematic uncertainty of the tagging acceptance. The dashed lines indicate the kinematic region used in this analysis. 


\section{ZEUS}
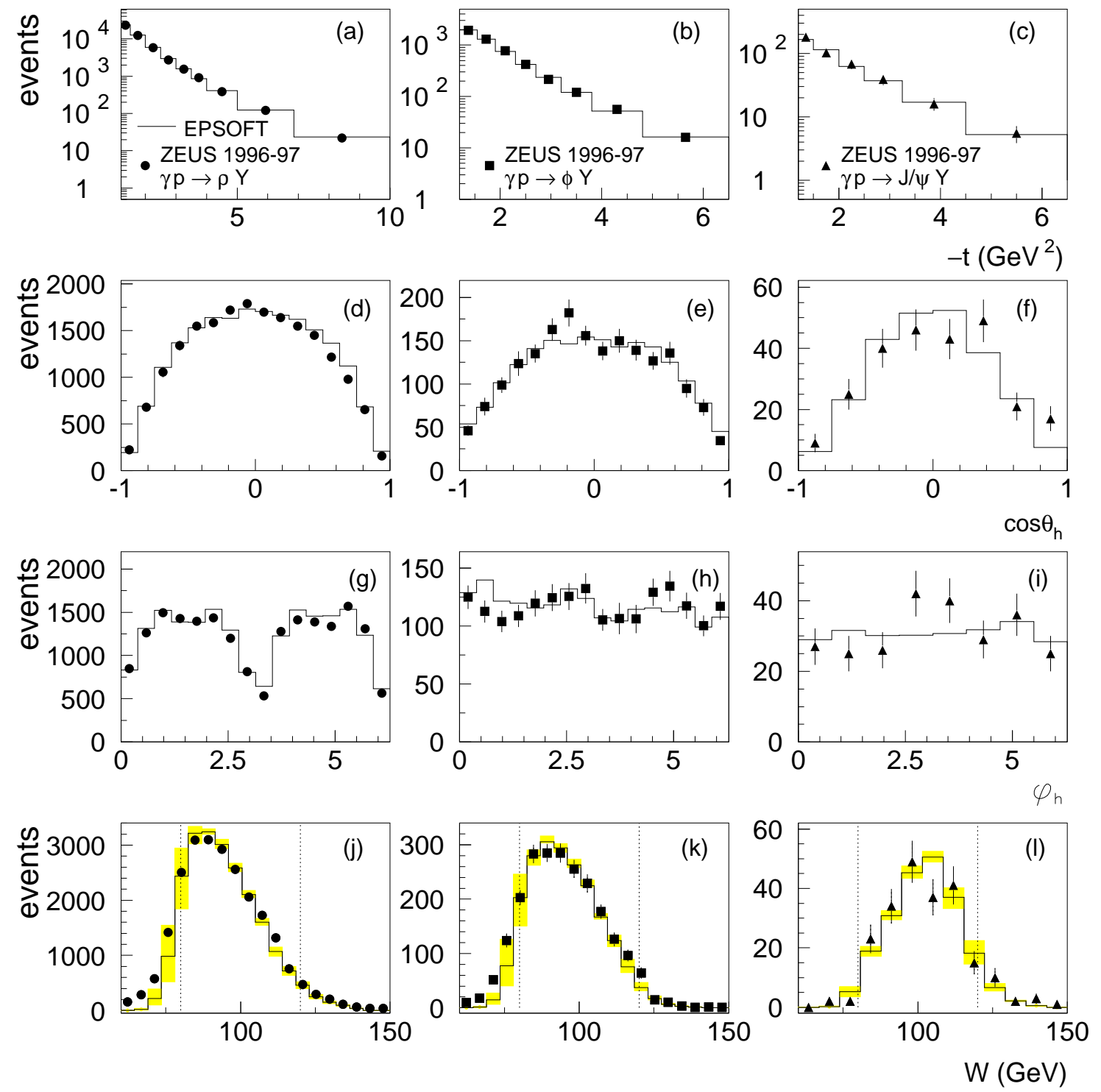

Figure 5: Comparison between the data and the EPSOFT MC (histograms) events for: $(a, b, c)-t ;(d, e, f) \cos \theta_{h} ;(g, h, i) \varphi_{h} ;(j, k, l) W$, after all selection cuts (except the cut on $W$ in the case of $W$ distributions). The $M C$ is normalised to the data. The dashed lines $(j, k, l)$ indicate the cuts on $W$ applied to select the final event sample. The systematic uncertainties due to the tagging acceptance are relevant in the case of $W$ distributions and are shown as the shaded bands. The three columns refer to the $\rho^{0}$, $\phi$, and $J / \psi$ samples, as indicated. 


\section{ZEUS}
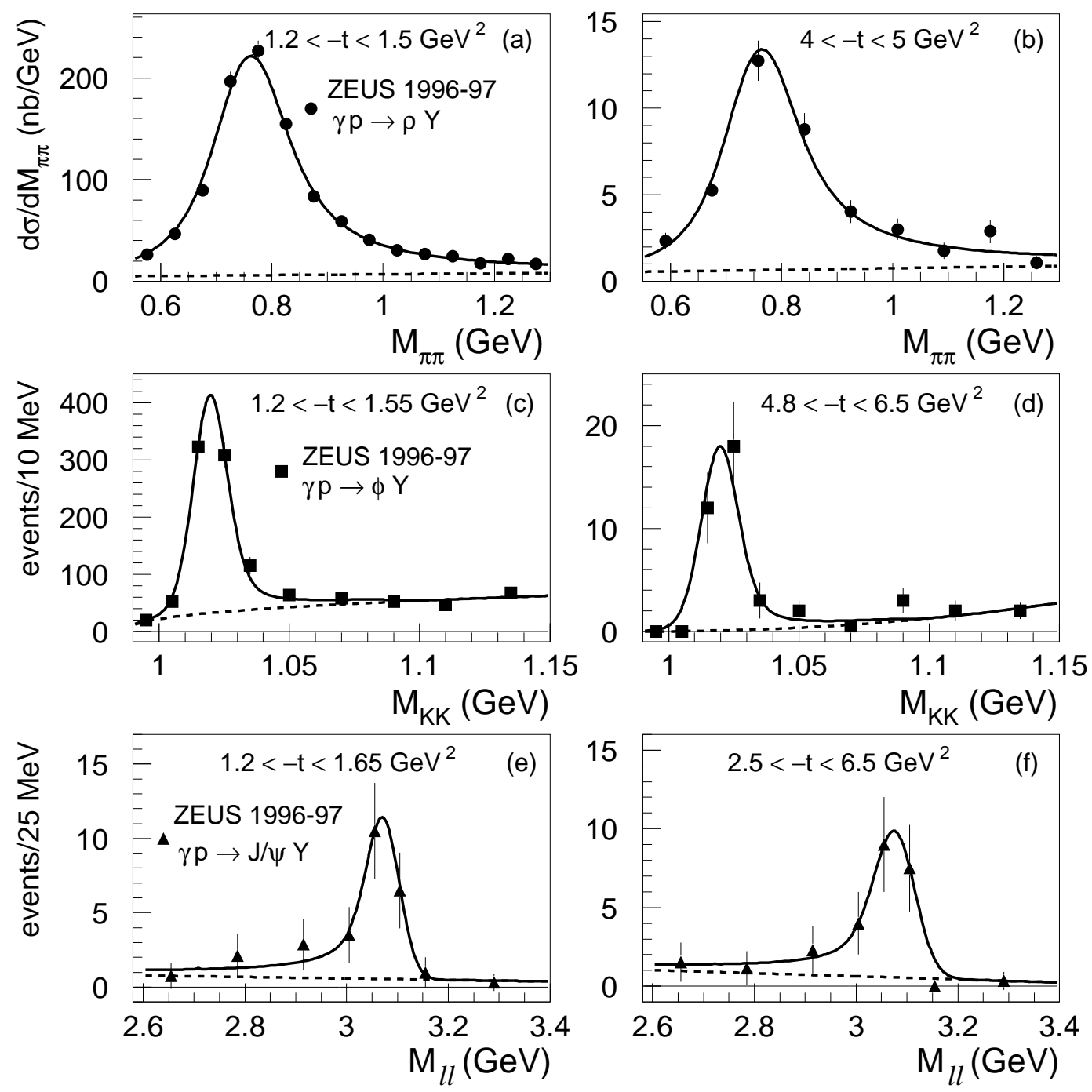

Figure 6: (a,b) The differential cross-section $\mathrm{d} \sigma / \mathrm{d} M_{\pi \pi}$ for the $\rho^{0}$ sample. $(c, d)$ The $M_{K K}$ mass distributions for the $\phi$ sample. (e,f) The $M_{l l}$ mass distributions for the $J / \psi$ sample. The symbols are the data for representative $t$ ranges and the solid curves indicate the result of the fits discussed in the text. The dashed curves show the background contributions. 
ZEUS

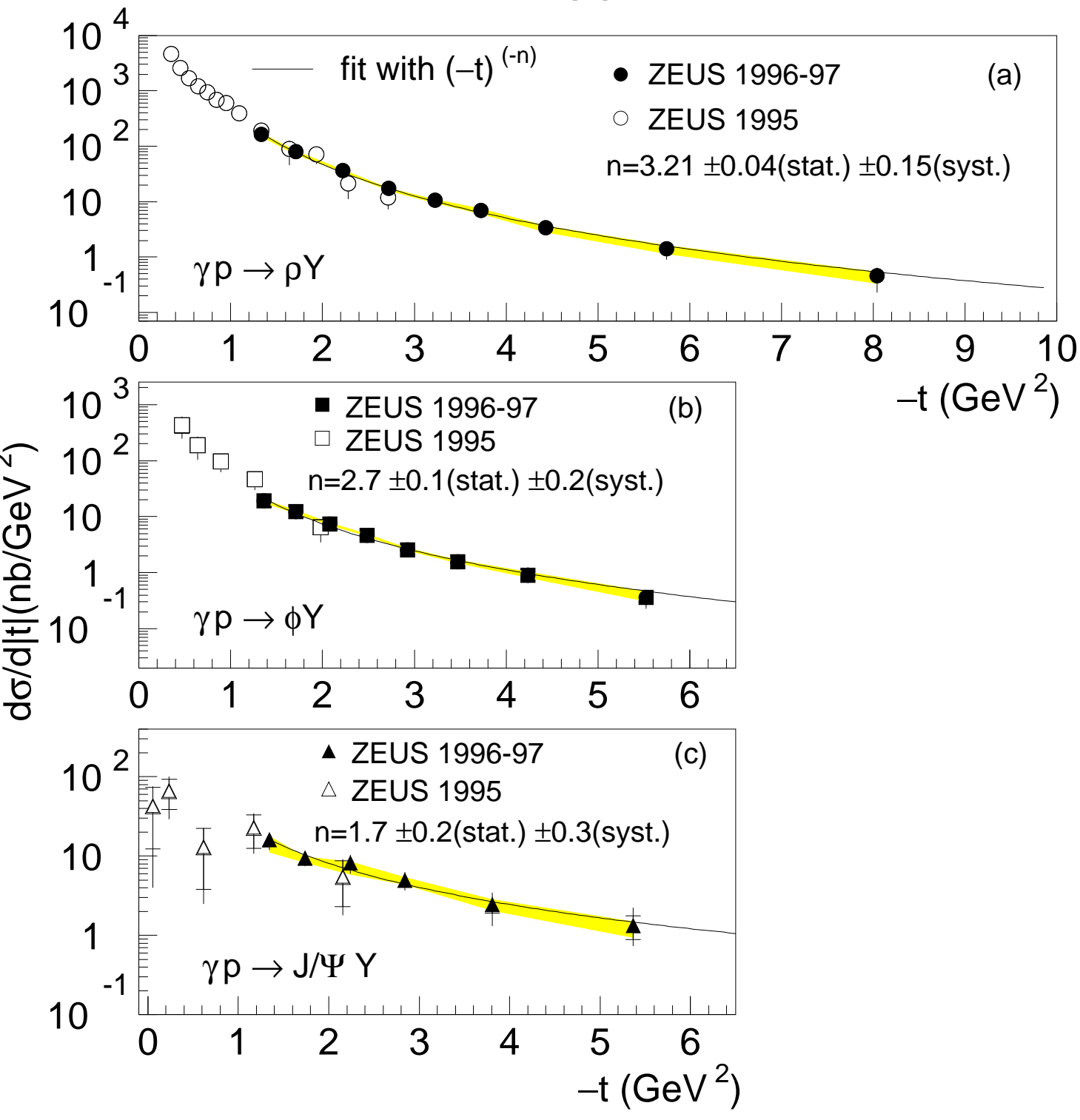

Figure 7: The differential cross sections $\mathrm{d} \sigma_{\gamma p \rightarrow V Y} / \mathrm{d}|t|$ in the range $80<W<$ $120 \mathrm{GeV}$ and $x>0.01$ for: (a) $\rho^{0}$, (b) $\phi$ and (c) $J / \psi$. The inner bars indicate the statistical uncertainty and the outer bars represent the statistical and systematic uncertainties added in quadrature. The shaded bands represent the correlated uncertainties due to the modelling of the hadronic-system $Y$. An additional correlated uncertainty of $\pm 10 \%$ is not shown. The open symbols correspond to the ZEUS 1995 results [10]. The lines are the results of the fit to the data with the function $A(-t)^{-n}$. 


\section{ZEUS}
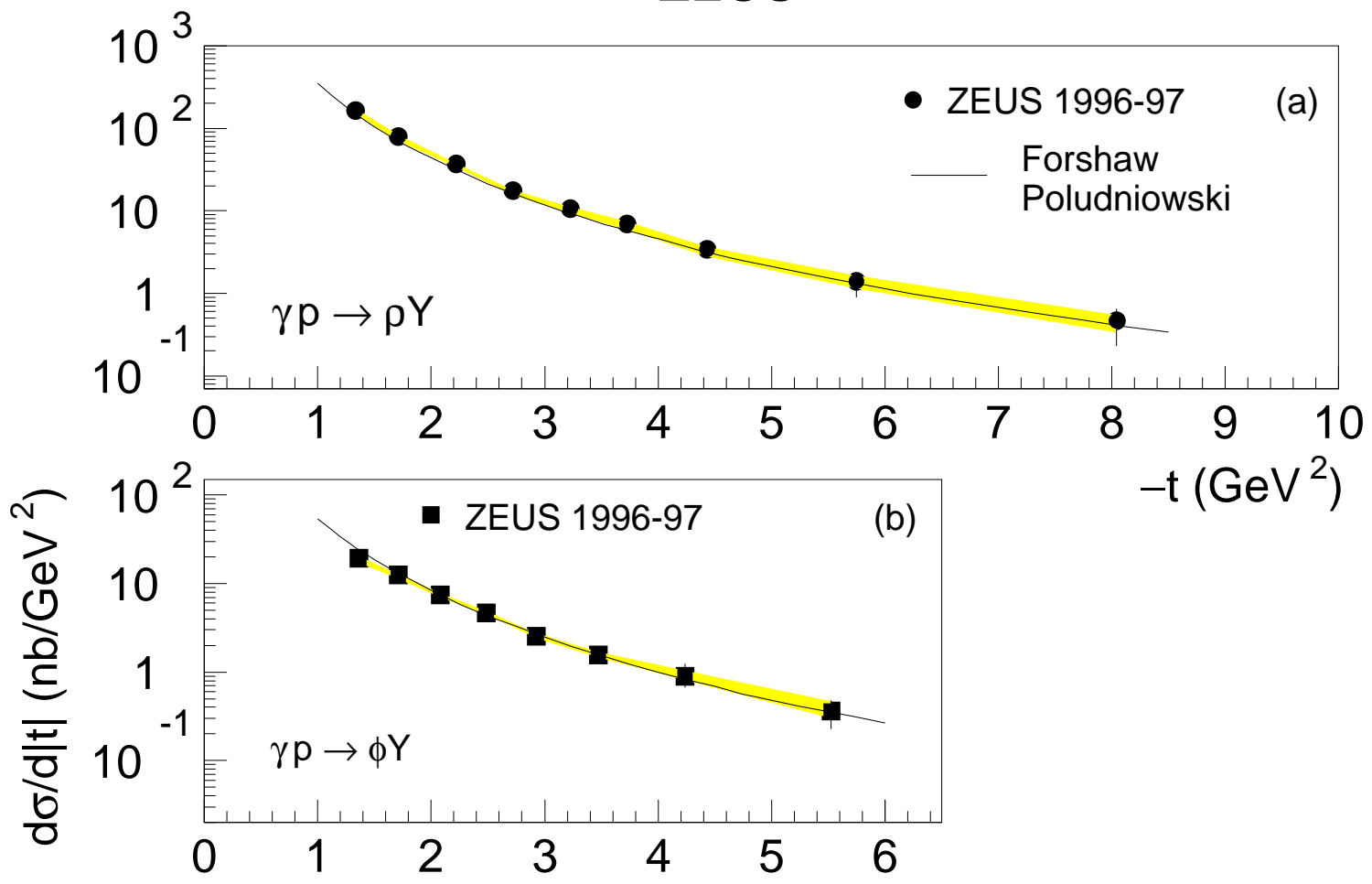

$-\mathrm{t}\left(\mathrm{GeV}^{2}\right)$

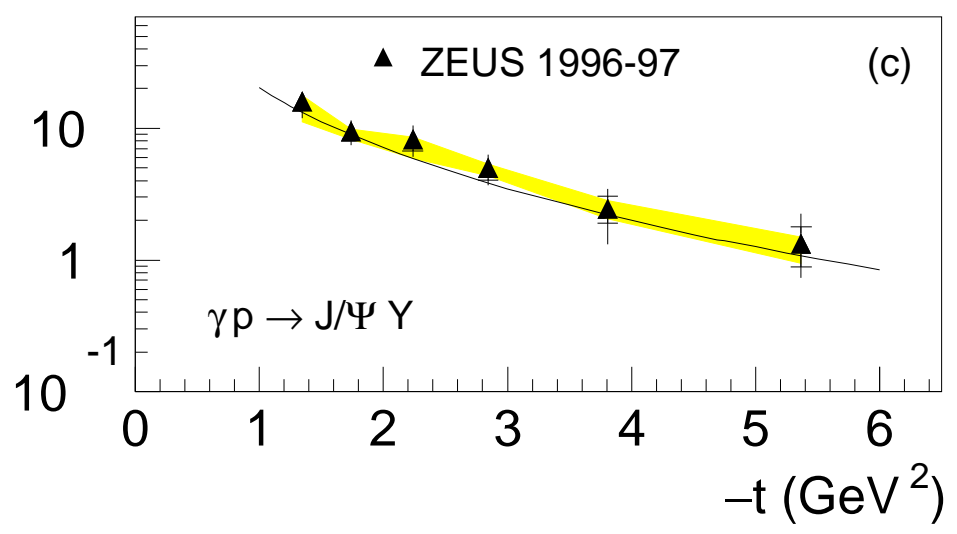

Figure 8: The differential cross sections $\mathrm{d} \sigma_{\gamma p \rightarrow V Y} / \mathrm{d}|t|$ for: (a) $\rho^{0}$, (b) $\phi$, and (c) $J / \psi$ photoproduction in the range $80<W<120 \mathrm{GeV}$ and $x>0.01$. The solid curves show a $p Q C D$ calculation [18]. The inner bars indicate the statistical uncertainty and the outer bars represent the statistical and systematic uncertainties added in quadrature. The shaded bands represent the size of the correlated uncertainties due to the modelling of the hadronic-system Y. An additional correlated uncertainty of $\pm 10 \%$ is not shown. 


\section{ZEUS}

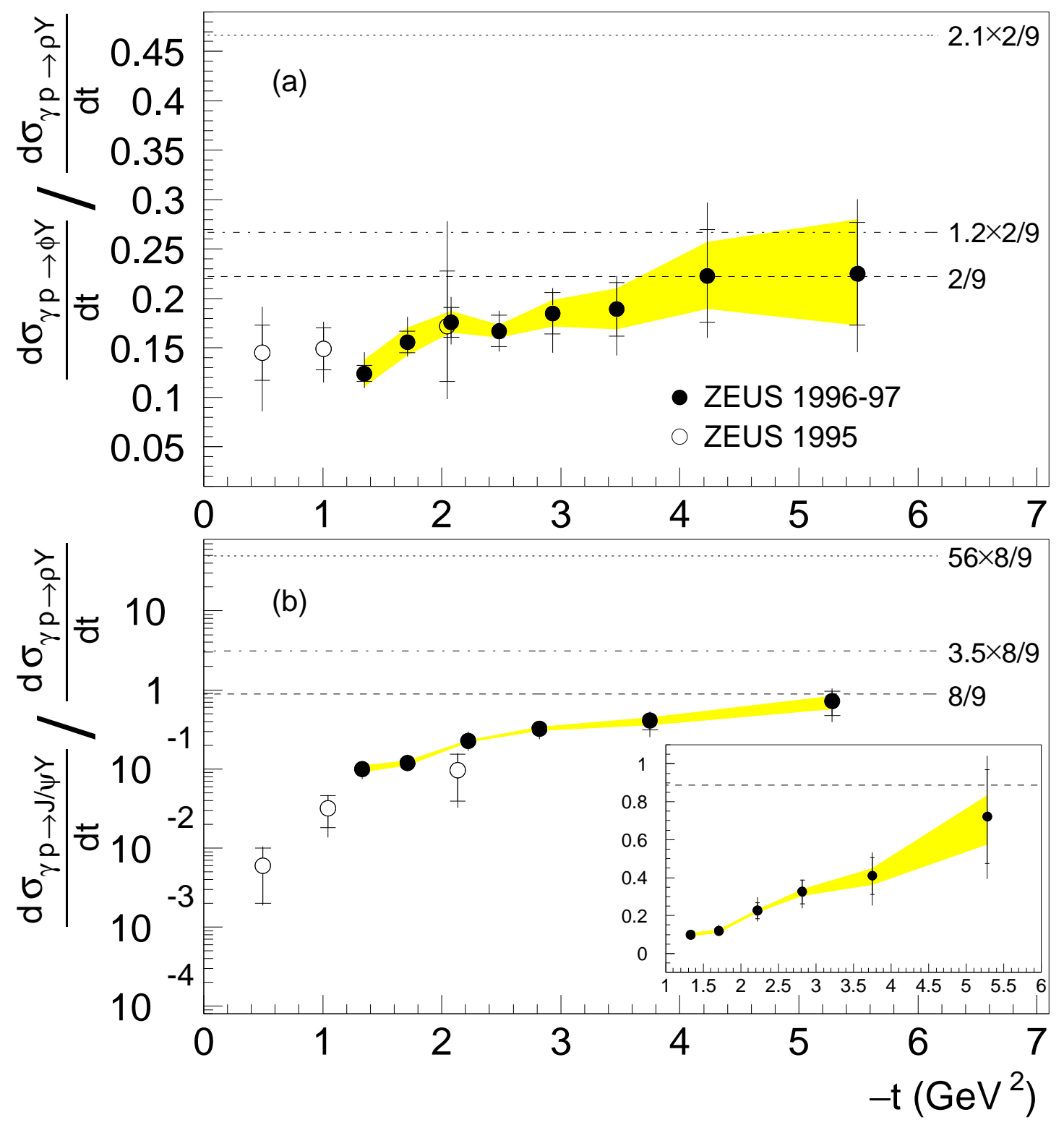

Figure 9: (a) The ratio of the cross sections $\mathrm{d} \sigma_{\gamma p \rightarrow V Y} / \mathrm{d} t$ for $\phi$ to $\rho^{0}$ photoproduction. (b) The ratio of the cross sections $\mathrm{d} \sigma_{\gamma p \rightarrow V Y} / \mathrm{d} t$ for $J / \psi$ to $\rho^{0}$ photoproduction. The inset shows this ratio on a linear scale. The inner bars indicate the statistical uncertainty, the outer bars represent the statistical and systematic uncertainties added in quadrature. The shaded bands represent the size of the correlated uncertainties due to the modelling of the dissociative system, $Y$. The dashed lines correspond to the $S U(4)$ predictions, while the dotted and dashed-dotted correspond to the $p Q C D$ values given by Eqs. (4) and (5), respectively. The open circles are the ZEUS 1995 results [10]. 


\section{ZEUS}

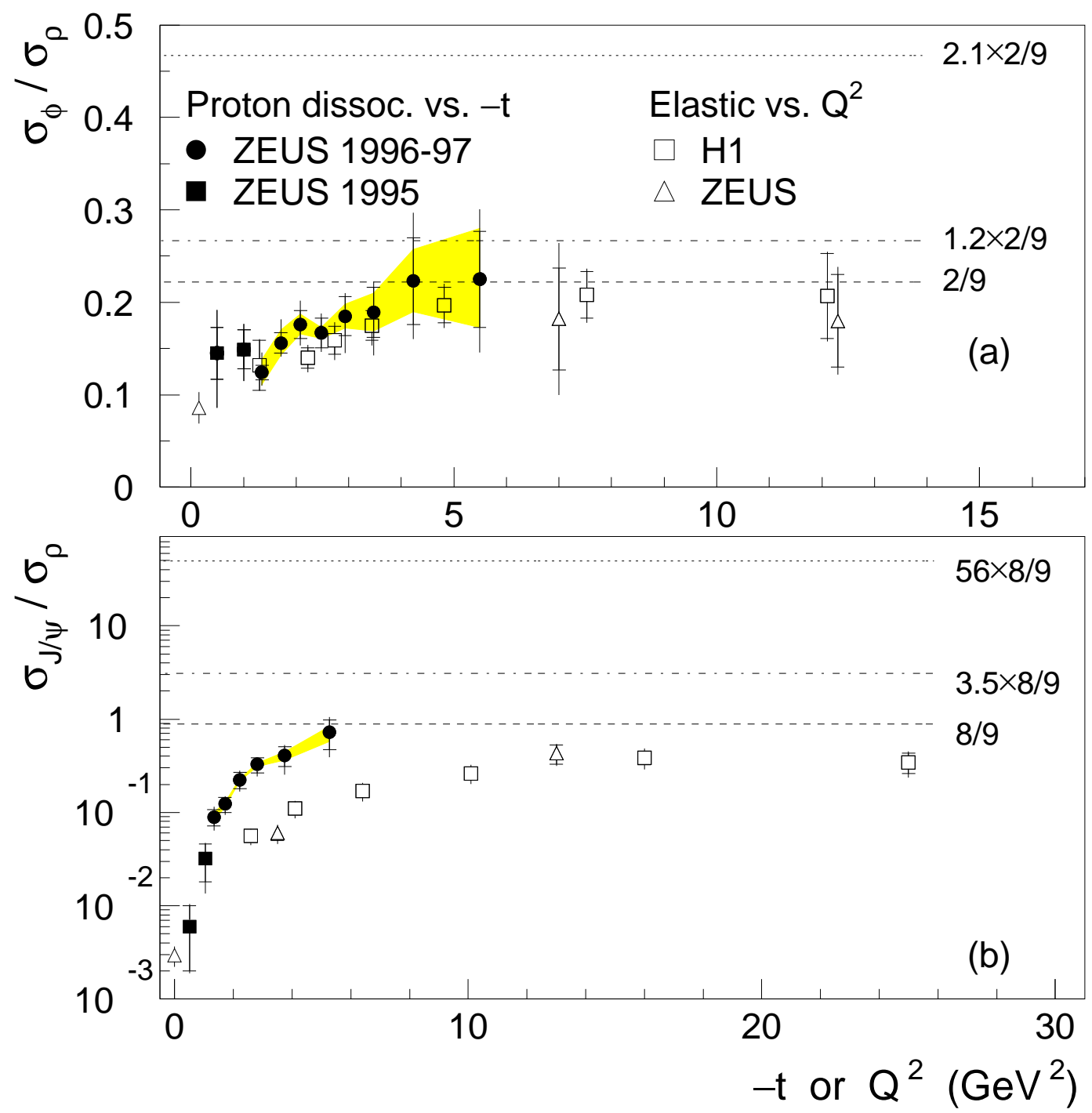

Figure 10: (a) The ratio of the $\phi$ to $\rho^{0}$ cross sections as a function of $-t$ or $Q^{2}$. The $\phi / \rho^{0}$ results as a function of $-t$ for proton-dissociative photoproduction from this analysis are shown with solid circles and those from the ZEUS 1995 [10] measurement with the solid squares. The shaded bands represent the size of the correlated uncertainties due to the modelling of the dissociative system, Y. Open triangles at $Q^{2} \approx 0 \mathrm{GeV}^{2}$ [49], $Q^{2}=7 \mathrm{GeV}^{2}$ [42] and $Q^{2}=12.3 \mathrm{GeV}^{2}$ [50] represent the $\phi / \rho^{0}$ ratio of the elastic cross sections as a function of $Q^{2}$ from ZEUS, while the open squares represent those from $H 1$ [41]. (b) The ratio of the $J / \psi$ to $\rho^{0}$ cross sections as a function of $-t$ or $Q^{2}$. The same convention for symbols as for $\phi / \rho^{0}$ ratio is used. Open triangles at $Q^{2} \approx 0 \mathrm{GeV}^{2}$ [51] and $Q^{2}=3.5,13 \mathrm{GeV}^{2}$ [43] represent the ZEUS measurements, while the open squares represent those of $H 1$ [44, 45]. The dashed lines correspond to the $S U(4)$ predictions, while the dotted and dashed-dotted correspond to the $p Q C D$ values given by Eqs. (4) and (5), respectively. 


\section{ZEUS}

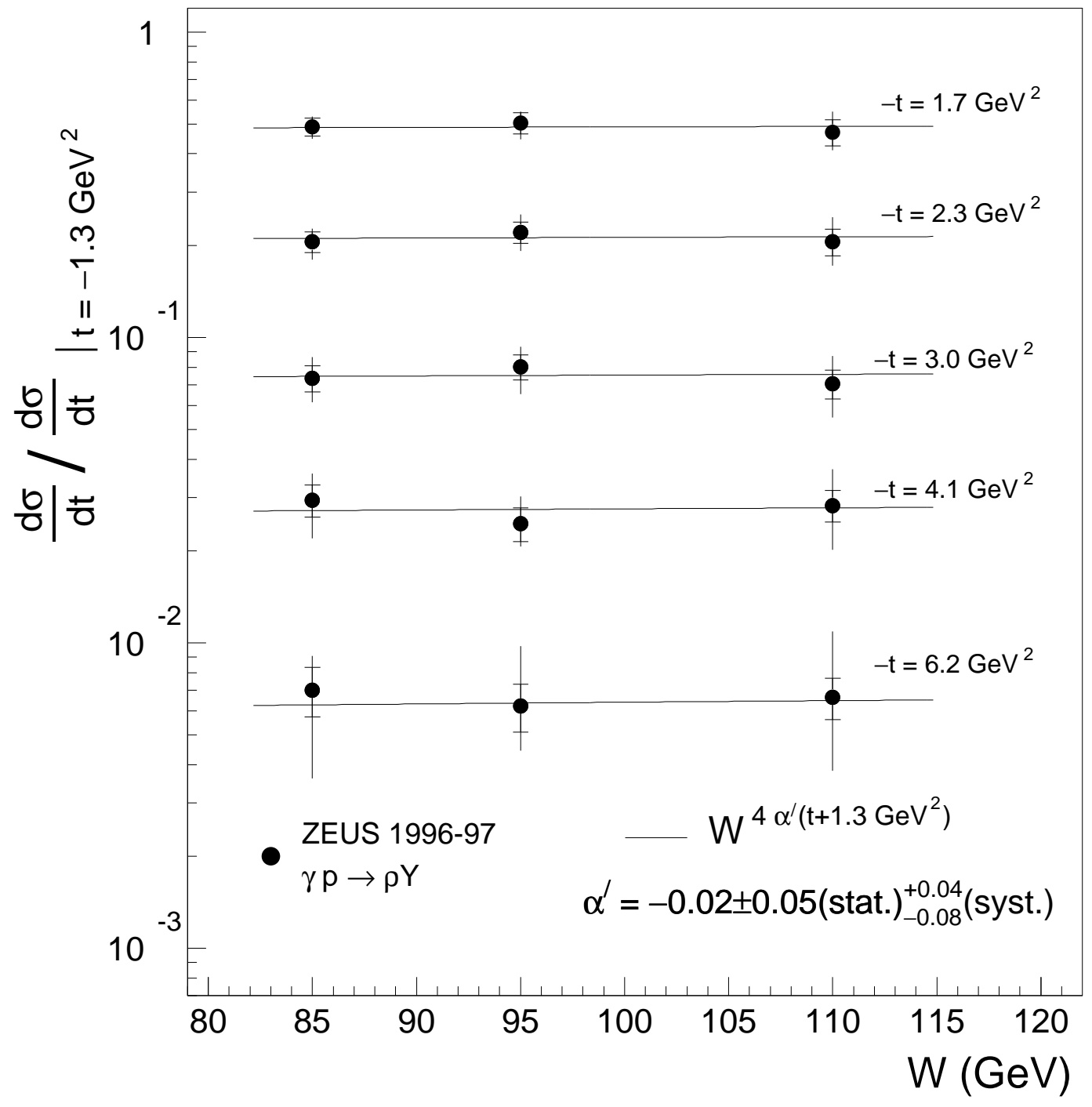

Figure 11: The ratios of $\rho^{0}$ production cross sections, $\frac{\mathrm{d} \sigma}{\mathrm{d} t} /\left.\frac{\mathrm{d} \sigma}{\mathrm{d} t}\right|_{t=t_{0}}$, for $-t_{0}=1.3$ $\mathrm{GeV}^{2}$, as a function of $W$ in five $t$ intervals. The lines represent the result of the fit with Eq. (19). The inner bars indicate the statistical uncertainty and the outer bars represent the statistical and systematic uncertainties added in quadrature. 


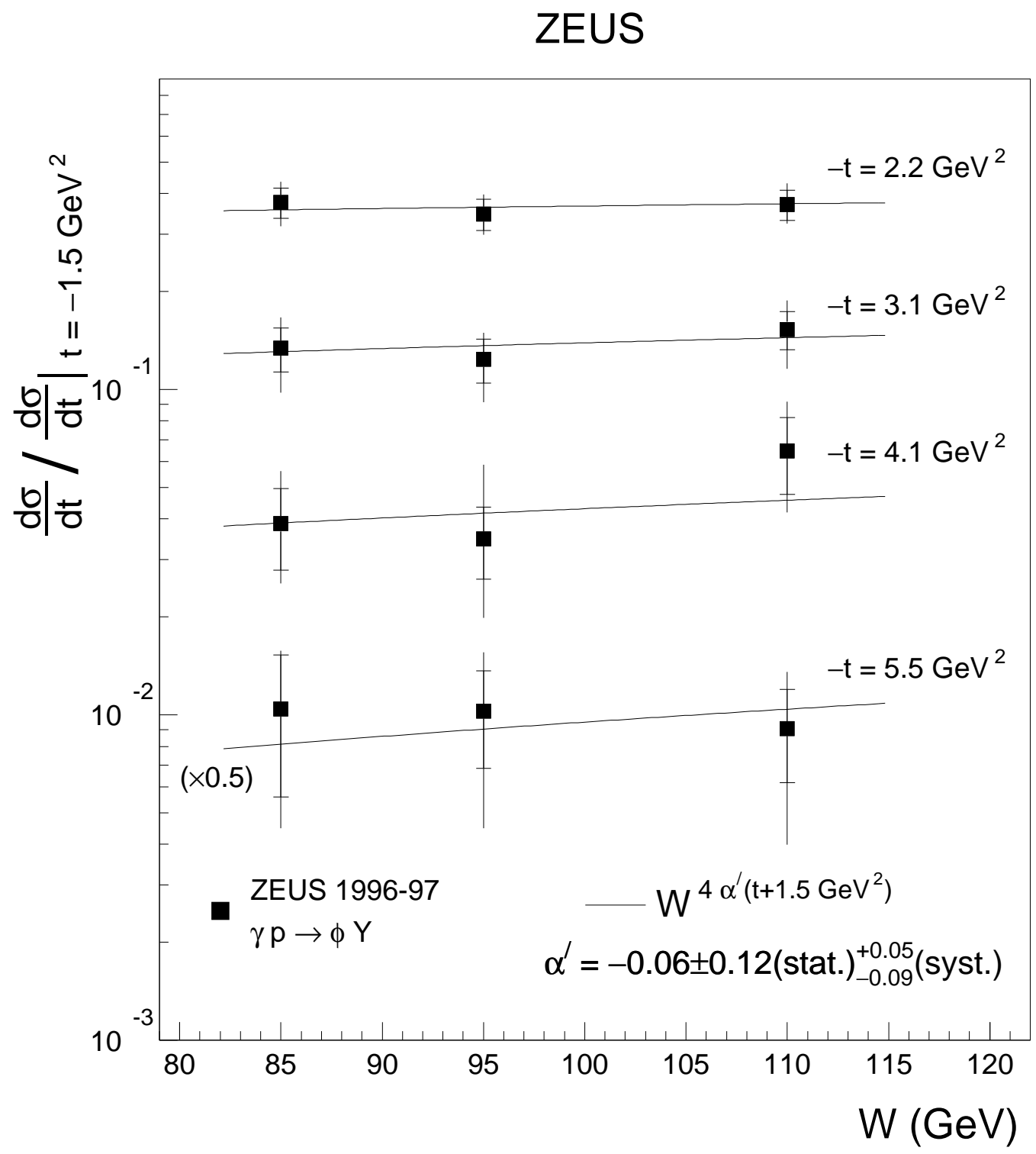

Figure 12: The ratios of $\phi$ production cross sections $\frac{\mathrm{d} \sigma}{\mathrm{d} t} /\left.\frac{\mathrm{d} \sigma}{\mathrm{d} t}\right|_{t=t_{0}}$, for $-t_{0}=1.5$ $\mathrm{GeV}^{2}$, as a function of $W$ in four $t$ intervals. The lines represent the result of the fit with Eq. (13). The inner bars indicate the statistical uncertainty and the outer bars represent the statistical and systematic uncertainties added in quadrature. 


\section{ZEUS}

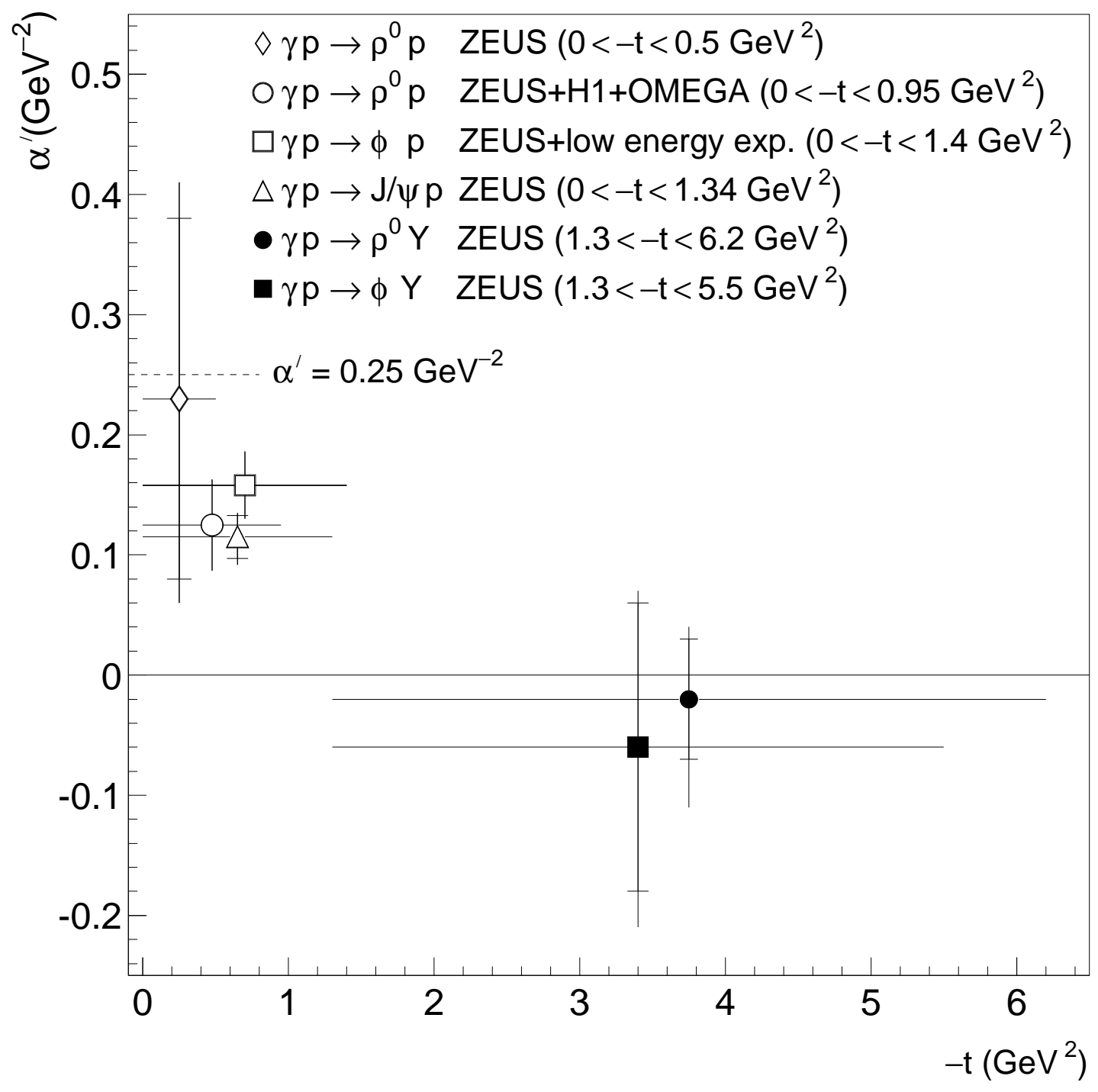

Figure 13: Comparison of results on $\alpha^{\prime}$ for vector-meson production. The result for the proton-dissociative photoproduction from this analysis are shown with solid symbols and those for elastic photoproduction with the open symbols. The value of $\alpha^{\prime}=0.25 \mathrm{GeV}^{-2}$ is characteristic of soft hadronic processes. The horizontal bars correspond to the $-t$ range in which $\alpha^{\prime}$ is measured. The vertical inner bars indicate the statistical uncertainty and the outer bars represent the statistical and systematic uncertainties added in quadrature. 


\section{ZEUS}
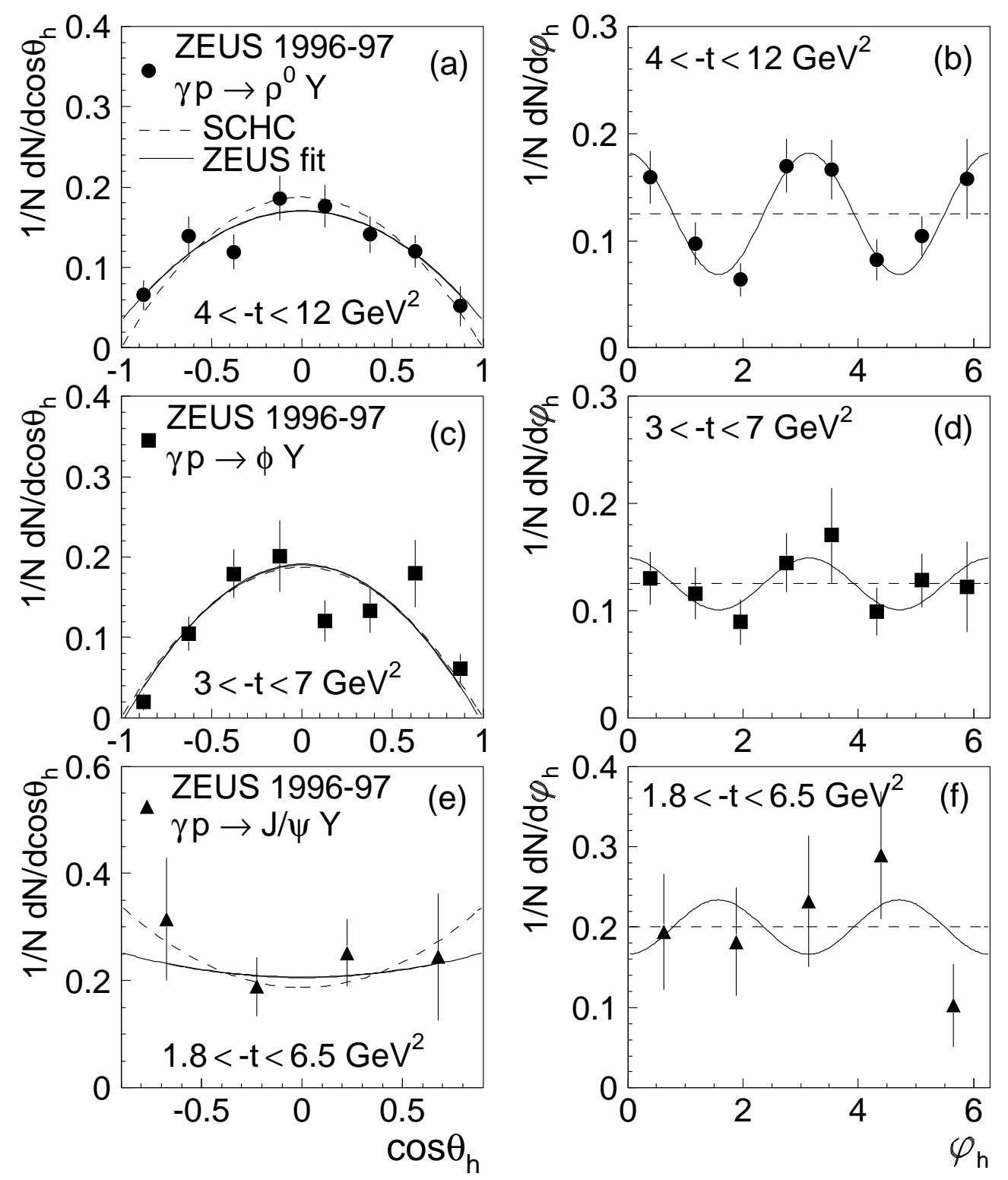

Figure 14: The normalised background-subtracted and acceptance-corrected $\cos \theta_{h}$ and $\varphi_{h}$ distributions for proton-dissociative photoproduction of: $(a, b) \rho^{0},(c, d) \phi$ and $(e, f) J / \psi$. The symbols represent the data and the solid curves the result of the fits with Eqs. (8) and (9). The dashed curves are the SCHC predictions. 


\section{ZEUS}

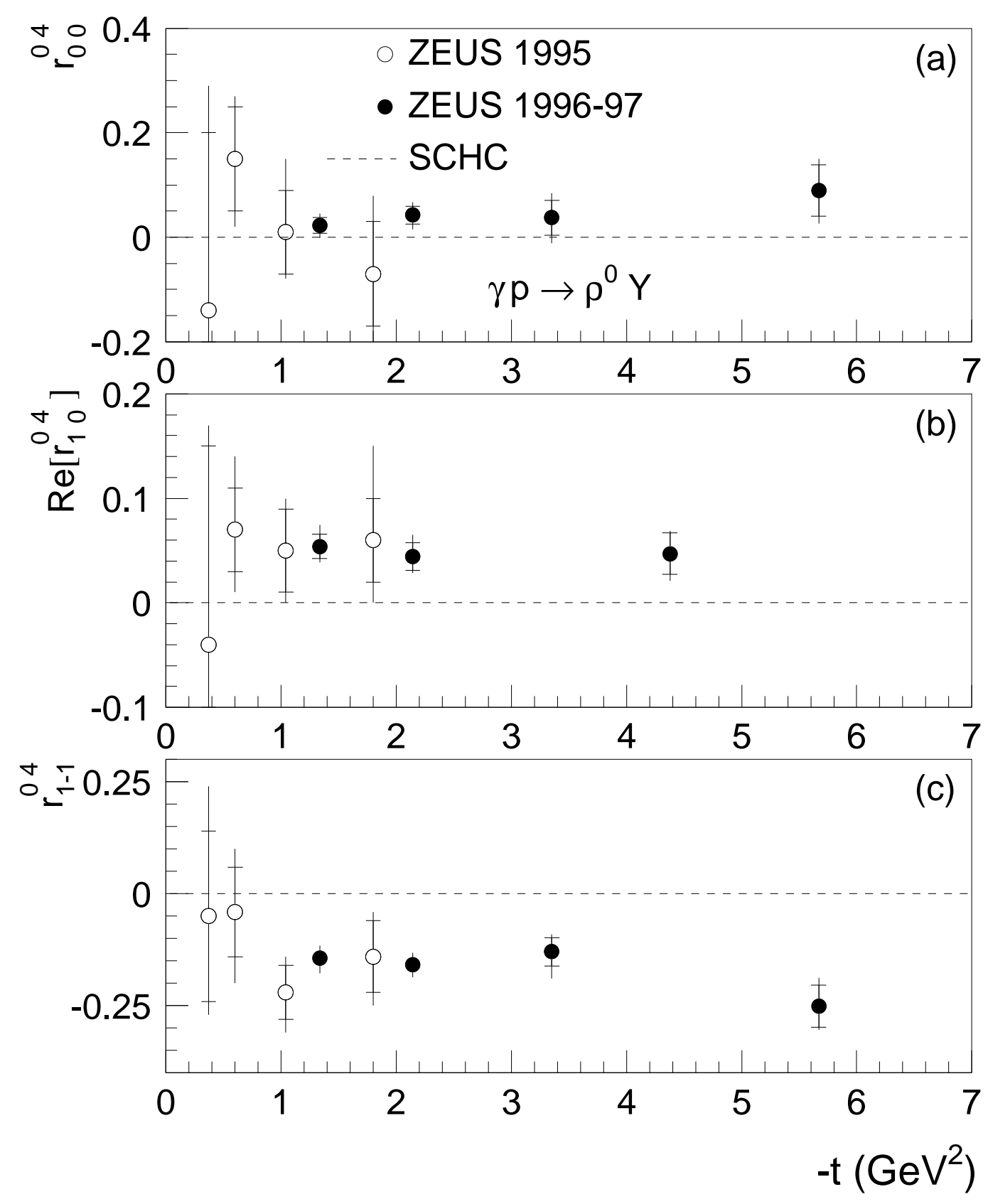

Figure 15: The fitted values of (a) $r_{00}^{04}$, (b) $R e\left[r_{10}^{04}\right]$ and (c) $r_{1-1}^{04}$ for protondissociative $\rho^{0}$ photoproduction as a function of $-t$. The inner bars indicate the statistical uncertainty and the outer bars represent the statistical and systematic uncertainties added in quadrature. The open circles correspond to the ZEUS 1995 results [10]. The SCHC prediction is shown as the dashed line. 


\section{ZEUS}

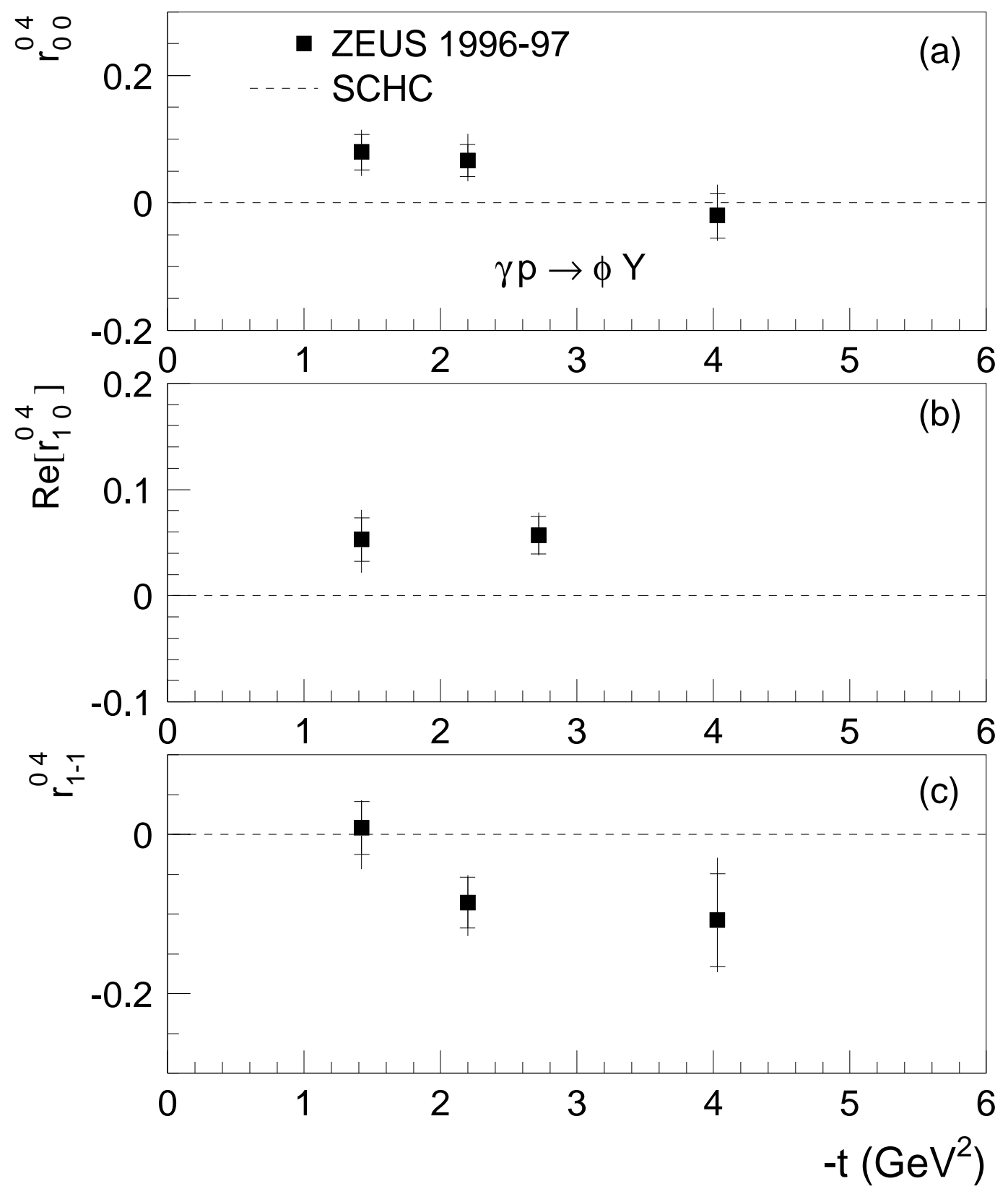

Figure 16: The fitted values of (a) $r_{00}^{04}$, (b) $R e\left[r_{10}^{04}\right]$ and (c) $r_{1-1}^{04}$ for protondissociative $\phi$ meson photoproduction as a function of $-t$. The inner bars indicate the statistical uncertainty and the outer bars represent the statistical and systematic uncertainties added in quadrature. The SCHC prediction is shown as the dashed line. 


\section{ZEUS}

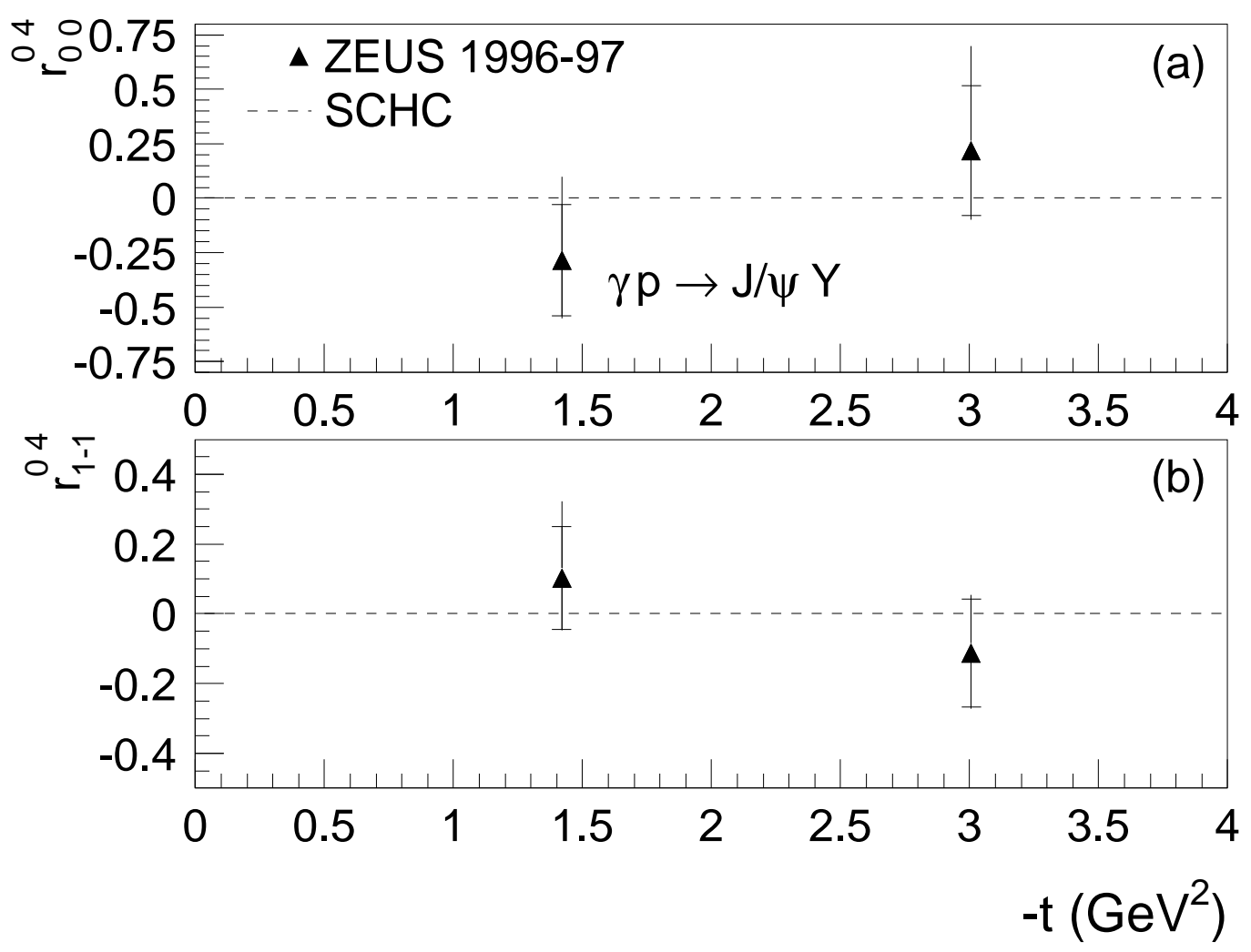

Figure 17: The fitted values of (a) $r_{00}^{04}$ and (b) $r_{1-1}^{04}$ for proton-dissociative $J / \psi$ meson photoproduction as a function of $-t$. The inner bars indicate the statistical uncertainty and the outer bars represent the statistical and systematic uncertainties added in quadrature. The SCHC prediction is shown as the dashed line. 